\title{
Dynamical similarity and instabilities in high-Stokes-number oscillatory flows of superfluid helium
}

\author{
D. Schmoranzer, ${ }^{1}$ M. J. Jackson, ${ }^{1}{ }^{S}$. Midlik, ${ }^{1}$ M. Skyba,,${ }^{2,}{ }^{*}$ J. Bahyl,${ }^{3}$ T. Skokánková, ${ }^{1}$ V. Tsepelin, ${ }^{4}$ and L. Skrbek ${ }^{1}$ \\ ${ }^{1}$ Faculty of Mathematics and Physics, Charles University, Ke Karlovu 3, 121 16, Prague 2, Czech Republic \\ ${ }^{2}$ Institute of Physics ASCR, v.v.i., Na Slovance 2, 182 21, Prague 8, Czech Republic \\ ${ }^{3}$ Faculty of Mathematics, Physics and Informatics, Comenius University, Mlynská dolina, 842 48, Bratislava, Slovakia \\ ${ }^{4}$ Physics Department, Lancaster University, Lancaster, LA1 4YB, United Kingdom
}

(Received 7 November 2018; published 19 February 2019)

\begin{abstract}
We present a unified analysis of the drag forces acting on oscillating bodies submerged in superfluid helium such as a vibrating wire resonator, tuning forks, a double-paddle oscillator, and a torsionally oscillating disk. We find that for high-Stokes-number oscillatory flows, the drag force originating from the normal component of superfluid helium exhibits a clearly defined universal scaling. Following classical fluid dynamics, we derive the universal scaling law and define relevant dimensionless parameters such as the Donnelly number. We verify this scaling experimentally using all of our oscillators in superfluid ${ }^{4} \mathrm{He}$ and validate the results by direct comparison with classical fluids. We use this approach to illustrate the transition from laminar to turbulent drag regime in superfluid oscillatory flows and compare the critical velocities associated to the production of quantized vortices in the superfluid component with the critical velocities for the classical instabilities occurring in the normal component. We show that depending on the temperature and geometry of the flow, either type of instability may occur first and we demonstrate their crossover due to the temperature dependence of the viscosity of the normal fluid. Our results have direct bearing on present investigations of superfluids using nanomechanical devices [Bradley et al., Sci. Rep. 7, 4876 (2017)].
\end{abstract}

DOI: 10.1103/PhysRevB.99.054511

\section{PREFACE}

Historically, experiments on oscillatory flows of classical viscous fluids have been studied since the days of Stokes [1], with many notable developments made in the last century [2-5]. Recently, oscillating flows have reemerged thanks to developments in micromechanical and nanomechanical engineering, where access to nano electromechanical systems (NEMS) [6-10] has offered unprecedented sensitivity and resolution in fluid dynamical experiments, allowing the transition from continuum to ballistic (molecular) regime to be probed at easily attainable pressures, directly probe fluid boundary layers [9], or formulate universality relations [6-8] for classical oscillatory flows. This work extends such universality relations to superfluids, concentrating on the hydrodynamic regime; the transitional and ballistic regimes will represent the subject of a later publication.

An extremely broad range of working fluids of well-known physical properties [11-13] may be obtained when traversing the different phases of helium, even limiting ourselves to the common isotope ${ }^{4} \mathrm{He}$. The normal liquid phase of ${ }^{4} \mathrm{He}$, known as He I, is a highly interesting working fluid thanks to its extremely low kinematic viscosity $v$, which provides

*Present address: Oxford Instruments, Abingdon, Oxfordshire, OX13 5QX, United Kingdom.

Published by the American Physical Society under the terms of the Creative Commons Attribution 4.0 International license. Further distribution of this work must maintain attribution to the author(s) and the published article's title, journal citation, and DOI. very high Reynolds number $\left(\operatorname{Re} \approx 10^{7}\right.$ ) flows in controlled laboratory experiments $[14,15]$. Similarly, cryogenic He gas provides extremely large Rayleigh numbers $\left(\mathrm{Ra} \approx 10^{17}\right)$ in convective flows [16]. Liquid ${ }^{4} \mathrm{He}$ undergoes a superfluid phase transition at $T_{\lambda} \approx 2.17 \mathrm{~K}$ at saturated vapor pressure. Superfluid ${ }^{4} \mathrm{He}$, or $\mathrm{He}$ II, is a quantum fluid, and its flow properties cannot be described by means of classical fluid dynamics. According to Landau's two-fluid model $[17,18]$, it behaves as if composed of two interpenetrating liquids (the normal and superfluid components) with individual velocity fields and temperature-dependent densities. At the superfluid transition at $T_{\lambda}$, the density of the normal component accounts for $100 \%$ of the total density, but drops rapidly with decreasing temperature and vanishes for $T \rightarrow 0 \mathrm{~K}$.

Oscillatory flows of He II have been studied using various oscillators such as disks [19,20], piles of disks [21], spheres [22-24], grids [25-29], tuning forks [30-33], reeds [34], double paddles [35-37], cylinders of rectangular [38] or circular cross-section (wires) [39-42] since the discovery of superfluidity, and have led to important insights to this fundamental physical phenomenon. For reviews, see [43,44]. Despite these efforts, a universal picture is still missing in superfluid hydrodynamics, which motivated us to investigate oscillatory flows of He II due to mechanical oscillators of largely varied geometries (vibrating tuning forks, a microwire loop, a torsionally oscillating disk, and a double-paddle oscillator) and search for universal features.

\section{INTRODUCTION}

In this section, we introduce the key concepts of superfluid hydrodynamics, and use classical oscillatory flows in 
the high-Stokes-number regime as a stepping stone to derive the properties of similar flows in superfluids. We limit our discussion of the dynamics of superfluids to the Newtoniantype hydrodynamic description applicable above $\simeq 1 \mathrm{~K}$, as this corresponds to most of the experiments described here.

\section{A. Superfluid hydrodynamics}

On a phenomenological level, superfluid ${ }^{4} \mathrm{He}$ at finite temperatures is described as consisting of two components: a viscous normal component and an inviscid superfluid component [17]. Their temperature-dependent densities $\rho_{\mathrm{n}}$ and $\rho_{\mathrm{s}}$, respectively, add up to the (nearly temperature-independent) total density of He II, $\rho$. While the normal component behaves classically, possessing finite viscosity and carrying the entire entropy content of He II, the superfluid component has neither entropy nor viscosity and, due to quantum restrictions, the vorticity is constrained into line singularities called quantized vortices [18]. In He II, each quantized vortex carries one quantum of circulation, given as $\kappa=h / m_{4} \approx 0.997 \times$ $10^{-7} \mathrm{~m}^{2} \mathrm{~s}^{-1}$, where $h$ is the Planck constant and $m_{4}$ denotes the mass of a ${ }^{4} \mathrm{He}$ atom. Superfluid turbulence [45] therefore takes the form of a dynamic tangle of quantized vortices in the superfluid component.

At temperatures above $\simeq 1 \mathrm{~K}$, where the fraction of the normal fluid is significant, this tangle of quantized vortices typically coexists with classical-like turbulent flow of the normal component, making up what is usually called quantum turbulence (turbulent flow of a quantum fluid). In the presence of quantized vortices, the otherwise independent normal and superfluid velocity fields become coupled by a mutual friction force which arises due to thermal excitations (responsible for the entropy and viscosity of the normal component) scattering off the cores of quantized vortices. Quantum turbulence can thus be loosely defined as the most general way of motion of quantum fluids displaying superfluidity [45].

Does quantum turbulence always contain quantized vortices? Strictly speaking, quantized vortices are not a necessary ingredient of quantum turbulence, as one can imagine a twofluid flow of He II consisting of turbulent normal flow and potential superflow. Indeed, in the hypothetical case of a macroscopic sample of He II free of quantized vortices (i.e., without mutual friction coupling the two velocity fields), in an isothermal flow the normal and superfluid components move independently and any instability criteria ought to be applied to them separately. In this hypothetical case, quantized vortices must be nucleated intrinsically; this process requires critical velocities of order $10 \mathrm{~m} / \mathrm{s}$ or higher [45]. In practice, however, remnant vortices always exist in macroscopic samples of He II and nucleation of quantized vorticity takes place extrinsically, by stretching and reconnections of seed vortex loops. In many types of flow, the critical velocity for extrinsic vortex nucleation is observed to be a few $\mathrm{cm} / \mathrm{s}$. As turbulence of the normal component may be possible even below this velocity threshold, it follows that there indeed is a possibility of having a quantum flow displaying (nearly) potential superflow together with a vortical flow of the normal component.

With very few remnant quantized vortices present, the mutual friction force is negligible and, according to the twofluid model of Landau [17], an isothermal flow of the normal component is described by the Navier-Stokes equations, while that of the superfluid component by the Euler equations for ideal fluids. Under these conditions, any body moving through He II at low velocity below the (generally independent) critical thresholds would experience drag forces originating from the normal component alone, while the drag forces offered by the superfluid component are zero (neglecting any drag due to surface waves and compressibility effects). In this case, the superfluid component can be understood as a physical vacuum, merely renormalizing the effective mass of the oscillating body by means of conservative inertial forces. Therefore, to derive the scaling laws for the drag forces in the Newtonian limit, we must analyze the Navier-Stokes equations governing the motion of the normal component.

\section{B. Classical oscillatory flows: Hydrodynamic limit}

To describe a classical oscillatory flow, the governing Navier-Stokes equations (NSE) may be expressed in terms of dimensionless velocity $\boldsymbol{u}^{\prime}=\boldsymbol{u} / U$, time $t^{\prime}=t / T$, and positions $\boldsymbol{r}^{\prime}=\boldsymbol{r} / L_{i}$ as

$$
\omega U \frac{\partial \boldsymbol{u}^{\prime}}{\partial t^{\prime}}+\frac{U^{2}}{L_{1}}\left(\boldsymbol{u}^{\prime} \cdot \nabla^{\prime} \boldsymbol{u}^{\prime}+\nabla^{\prime} p^{\prime}\right)=\frac{\nu U}{L_{2}{ }^{2}} \Delta^{\prime} \boldsymbol{u}^{\prime},
$$

where the characteristic length scales $L_{1,2}$ are used together with the characteristic velocity $U$ to estimate the maximum magnitude of the respective velocity derivatives. An independent timescale is introduced, given (in the continuum limit) by the angular frequency of oscillation $\omega$. Generally, the choice of $L_{1}$ and $L_{2}$ depends on body geometry and flow parameters. Candidates may include the typical body size $D$, the surface roughness $R_{q}$, or the Stokes boundary layer thickness (viscous penetration depth), defined as $\delta=\sqrt{2 \eta /(\rho \omega)}$, where $\eta$ denotes the dynamic viscosity of the working fluid. If, for a given body $\delta \ll D$, one may say that the object oscillates in the high-frequency regime, which is equivalent to the highStokes-number limit $\mathrm{St}=D^{2} /\left(\pi \delta^{2}\right) \gg 1$.

In the high-frequency limit, depending on body geometry (especially surface roughness and the presence of sharp corners), $\delta$ or $D$ may take the part of $L_{1}$ (related to the largest tangential velocity derivative) in the NSE, but it is always $\delta$ that takes the part of $L_{2}$ (related to the largest velocity derivative in any direction) (see Fig. 1). When sharp corners are present [case (a) in Fig. 1] or when $R_{q} \gg \delta$ [case (d) in Fig. 1], one may safely put $L_{1}=L_{2}=\delta$, and the Navier-Stokes equation may be expressed using only one dimensionless parameter, the boundary-layer-based Reynolds number: $\operatorname{Re}_{\delta} \equiv(\delta \rho U) / \eta$. Conversely, for a hydrodynamically smooth body $\left(R_{q} \ll \delta\right)$ without any sharp corners, such as a cylinder [case (b) in Fig. 1], one would obtain the Navier-Stokes equation with the Keulegan-Carpenter number $\mathrm{K}_{\mathrm{C}}=U T / D$ as the only relevant dimensionless parameter [2]. However, for laminar flows with $\mathrm{K}_{\mathrm{C}} \ll 1$, where the nonlinear term can be neglected, the viscous drag force would still be expected to scale with $\operatorname{Re}_{\delta}$, as for the viscous drag $L_{2}=\delta$ is the only relevant length scale even in this case.

Of the oscillators used in this work, only the disk may be considered hydrodynamically smooth. The classification of our oscillators would thus be tuning forks [Figs. 1(a) and 1(d)], vibrating wire [Fig. 1(d)], double paddle [Fig. 1(a)], 

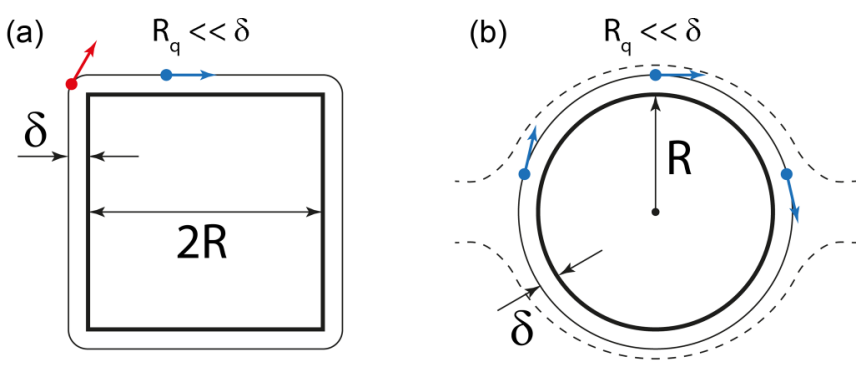

(c)

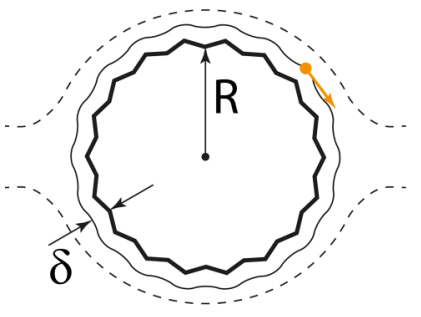

$\mathrm{R}_{\mathrm{q}} \sim \delta$

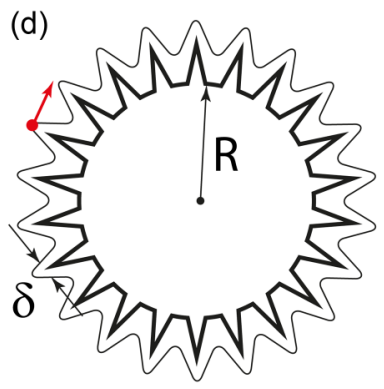

$\mathrm{R}_{\mathrm{q}}>>\delta$

FIG. 1. Illustration of the effects of surface roughness and sharp corners on the estimates of (maximum values of) velocity derivatives in the high-frequency limit, where $\delta \ll R$. In cases (a) and (d), both velocity derivatives present in the NSE are estimated using $\delta$, while in case (b), the tangential velocity derivative is estimated using $R$ and the Laplacian using $\delta$. In case (c), where $R_{q} \simeq \delta$, reliable estimates of the tangential derivative prove difficult; a smooth crossover between cases (b) and (d) is expected.

disk [Fig. 1(b)]; see Sec. III for details. The tuning forks contain sharp corners and have a surface roughness exceeding the boundary-layer thickness at the same time. We expect the roughness to be more significant for the commercial tuning fork (see Sec. III A).

\section{Oscillatory flows of He II}

Assuming two independent velocity fields in He II, as is the case at low velocities, where the normal component exhibits laminar flow and the superfluid component remains potential, the above considerations are fully applicable to the oscillatory viscous flow of the normal component. We therefore replace $\rho$ by $\rho_{\mathrm{n}}$, decompose the pressure into partial pressures of the normal and superfluid components, and replace $\delta$ by $\delta_{\mathrm{n}}=$ $\sqrt{2 \eta /\left(\rho_{\mathrm{n}} \omega\right)}$, where $\eta$ denotes the dynamic viscosity of $\mathrm{He}$ II. Again, if, for a given body $\delta_{\mathrm{n}} \ll D$, and $R_{q} \gg \delta_{\mathrm{n}}$ (in our experiments, typically $\delta_{\mathrm{n}} \approx 1 \mu \mathrm{m}$, except for the disk, where $\delta_{\mathrm{n}} \approx 0.5 \mathrm{~mm}$ ), we may put $L_{1}=L_{2}=\delta_{\mathrm{n}}$, and the NavierStokes equation may be written using only one dimensionless parameter: Dn $\equiv\left(\delta_{\mathrm{n}} \rho_{\mathrm{n}} U\right) / \eta$, which we call the Donnelly number [46]:

$$
2 \frac{\partial \boldsymbol{u}^{\prime}}{\partial t^{\prime}}+\operatorname{Dn}\left(\boldsymbol{u}^{\prime} \cdot \nabla^{\prime} \boldsymbol{u}^{\prime}+\nabla^{\prime} p_{\mathrm{n}}^{\prime}\right)=\Delta^{\prime} \boldsymbol{u}^{\prime} .
$$

We note that Dn will become equivalent to $\operatorname{Re}_{\delta}$ at the superfluid transition temperature $T_{\lambda}$, allowing direct comparison with classical fluids.

If $\delta_{\mathrm{n}} \ll R$ is satisfied (high-Stokes-number limit), then the flow may be regarded as potential everywhere outside the thin boundary layer of thickness on the scale of $\delta_{\mathrm{n}}$. Moreover, if $\delta_{\mathrm{n}}$ is smaller than the typical radius of curvature of the oscillator surface, the surface may be described as consisting of many planar elements, and the velocity profile within the boundary layer is given by the solution to Stokes second problem (an oscillating plane). In laminar flow around such a body, the average energy dissipation per unit time is given by [47]

$$
\langle\dot{E}\rangle=\frac{1}{2} \frac{\eta}{\delta_{\mathrm{n}}} \oint\left|\Delta v_{\mathrm{L} 0, \mathrm{t}}\right|^{2} d S=\frac{1}{2} \frac{\eta}{\delta_{\mathrm{n}}} \oint \alpha_{\mathrm{L}}^{2} u_{\mathrm{L} 0}^{2} d S,
$$

where $\Delta v_{\mathrm{L} 0, \mathrm{t}}$ is the difference between two local velocity amplitudes projected tangentially to the surface, that of the potential flow just outside the boundary layer and that of the surface element of the body. Then, $\alpha_{\mathrm{L}}$ is the local flow enhancement factor relating this velocity difference to the (local) velocity amplitude $u_{\mathrm{L} 0}$ of the surface element in question: $\left|\Delta v_{\mathrm{L} 0, \mathrm{t}}\right|=\alpha_{\mathrm{L}} u_{\mathrm{L} 0}$. Integrating over the entire surface of an oscillator, we get

$$
\langle\dot{E}\rangle=\frac{\alpha \xi U_{\mathrm{p}}^{2} S_{\mathrm{r}}}{2} \frac{\eta}{\delta_{\mathrm{n}}},
$$

where $U_{\mathrm{p}}$ is the maximum velocity amplitude along the surface of the resonator (peak velocity). The dimensionless quantity of order unity $\xi=\oint u_{\mathrm{L}}^{2} d S /\left(S_{\mathrm{r}} U_{\mathrm{p}}^{2}\right)$ describes the velocity profile along the resonator, and an effective surface area $S_{\mathrm{r}} \geqslant$ $S$ may be used to account approximately for surface roughness. The integrated flow enhancement factor $\alpha$ is defined from $\alpha \xi=\oint \alpha_{\mathrm{L}}^{2} u_{\mathrm{L} 0}^{2} d S /\left(S_{\mathrm{r}} U_{\mathrm{p}}^{2}\right)$. We note that for a smooth rigid oscillator this becomes $\alpha=\oint \alpha_{\mathrm{L}}^{2} d S / S$, e.g., for a sphere: $\alpha_{\mathrm{L}}=3 / 2 \sin (\theta)$, with the angle $\theta$ measured from the direction of the flow, and $\alpha=\frac{3}{2}$. Similarly, for a cylinder oriented normally to flow, $\alpha_{\mathrm{L}}=2 \sin (\theta)$ and $\alpha=2$. We emphasize that the above derivation is valid for all the cases described in Fig. 1, as the length scale relevant to viscous drag is always $\delta_{n}$.

Using the peak velocity $U_{\mathrm{p}}$, it is possible to model a given mode of the resonator as a one-dimensional (1D) linear harmonic oscillator, as done in Ref. [30] for a tuning fork. This leads to the definition of a (net) dissipative force amplitude:

$$
F=\frac{2\langle\dot{E}\rangle}{U_{\mathrm{p}}}=\frac{\alpha \xi \eta}{\delta_{\mathrm{n}}} S_{\mathrm{r}} U_{\mathrm{p}} .
$$

We note that this force is meaningful only in the 1D model of the given resonant mode (or for a rigid oscillator) and does not, generally, offer a direct measure of the total forces experienced by the body. In analogy with steady flow, we define the dimensionless drag coefficient related to the normal component of He II as

$$
C_{\mathrm{D}}^{\mathrm{n}}=\frac{2 F}{A \rho_{\mathrm{n}} U_{\mathrm{p}}^{2}}=\frac{2 \alpha \xi S_{\mathrm{r}}}{A} \frac{\eta}{\rho_{\mathrm{n}} U_{\mathrm{p}} \delta_{\mathrm{n}}} \equiv \Phi / \mathrm{Dn},
$$

where $A$ is the sectional area perpendicular to the direction of flow, and the dimensionless quantity $\Phi=2 \alpha \xi S_{\mathrm{r}} / A$ is determined purely by the geometry of the oscillator. This scaling law is valid universally for laminar flow around all types of objects shown in Fig. 1.

Additionally, in accordance with the principle of dynamical similarity, for hydrodynamically rough bodies or bodies with sharp corners, the normal fluid drag coefficient may be expressed as a unique function of the Donnelly number 
$C_{\mathrm{D}}^{\mathrm{n}}=C_{\mathrm{D}}^{\mathrm{n}}(\mathrm{Dn})$ even in nonlaminar flow. Any departure from this function must then signify either a violation of these assumptions or an instability occurring in the superfluid component. In such a case, if the superfluid component becomes turbulent at some critical velocity $U_{\mathrm{C}}$, we expect a marked increase in the drag coefficient above the dependence $C_{\mathrm{D}}^{\mathrm{n}}(\mathrm{Dn})$ measured in a classical fluid (substituting the total density $\rho$ for $\rho_{\mathrm{n}}$ and $\operatorname{Re}_{\delta}$ for $\left.\mathrm{Dn}\right)$.

The Donnelly-Glaberson (DG) instability leading to the production of quantized vorticity in the superfluid is related to self-reconnections of seed vortex loops. This process has been described in the literature [48,49], and for macroscopic objects, the related critical velocity is expected to scale as $U_{\mathrm{C}} \propto \sqrt{\kappa \omega}$. Hence, it is convenient to define a reduced dimensionless velocity $\hat{U}=U_{\mathrm{p}} / \sqrt{\kappa \omega}$. To facilitate a hydrodynamic description of the drag forces originating in the superfluid component, we also define the superfluid drag coefficient

$$
C_{\mathrm{D}}^{\mathrm{s}}=\frac{2 F}{A \rho_{\mathrm{s}} U_{\mathrm{p}}^{2}}=\frac{2 F}{A \rho_{\mathrm{s}} \kappa \omega \hat{U}^{2}} .
$$

For laminar/potential flow of normal/superfluid components, this reduces to

$$
C_{\mathrm{D}}^{\mathrm{s}}=\frac{\phi}{\hat{U}} ; \quad \phi=\Phi \sqrt{\frac{\eta \rho_{\mathrm{n}}}{2 \kappa \rho_{\mathrm{s}}^{2}}},
$$

where $\Phi$ is the same as above. If turbulence is triggered in the superfluid component without any significant coupling to the normal component, again a unique function $C_{\mathrm{D}}^{\mathrm{s}}(\hat{U})$ should be observed. However, this scenario seems unlikely except close to the critical velocity, as the action of the mutual friction force would couple the two components when a sufficient density of quantized vortices is produced.

In the turbulent drag regime, at velocities sufficiently above the critical values, the normal and superfluid components are expected to be coupled due to the mutual friction force and contribute to the pressure drag together. In this situation, the classical definition of the drag coefficient is applicable: $C_{\mathrm{D}}=$ $2 F /\left(A \rho U^{2}\right)$, where the total density $\rho=\rho_{\mathrm{n}}+\rho_{\mathrm{s}}$ is used. It is expected that in coupled turbulent flows, $C_{\mathrm{D}}$ will tend towards a temperature-independent constant value of order unity $[43,50]$.

The total energy contained in the oscillatory motion of the resonator and the fluid is given as $E=m_{\mathrm{eff}} U_{p}^{2} / 2$, defining the effective mass of the resonant mode $m_{\mathrm{eff}}$. For a quasi-one- or two-dimensional resonator oscillating perpendicularly to its large dimension(s), such as a thin cantilever, beam, or membrane, it follows that $m_{\mathrm{eff}}=\xi m+m_{\mathrm{HD}}$, where $m$ is the actual mass of the resonator and $m_{\mathrm{HD}}$ represents the hydrodynamic added mass. If the hydrodynamic mass contribution can be neglected, it is convenient to define a fluidic quality factor $Q_{\mathrm{f}}$ :

$$
\frac{1}{Q_{\mathrm{f}}} \equiv \frac{\langle\dot{E}\rangle}{\omega E}=\frac{\alpha \xi S_{\mathrm{r}}}{m_{\mathrm{eff}}} \sqrt{\frac{\eta \rho_{\mathrm{n}}}{2 \omega}} \approx \frac{\alpha \rho_{\mathrm{n}} S_{\mathrm{r}} \delta_{\mathrm{n}}}{2 m},
$$

which can be directly linked to the resonant frequency $f$ and linewidth $\Delta$ by $Q_{\mathrm{f}}=f /\left(\Delta-\Delta_{0}\right)$, where $\Delta_{0}$ is the linewidth in vacuum. Conversely, the effective mass may be expressed from the resonant frequency in vacuum $f_{0}$ as $m_{\text {eff }} /(\xi m)=$ $\left(f_{0} / f\right)^{2}$.

The fluidic quality factor in Eq. (9) differs from the one given in Ref. [7] (in the limit of Newtonian hydrodynamics) by the explicit inclusion of the flow enhancement factor $\alpha$. We note that this factor is related to the potential flow outside the boundary layer and is necessary not only to recover correctly the analytical solutions obtained for the drag force acting on an oscillating sphere or cylinder, but in fact for all oscillators with nontrivial geometry. The fluidic quality factor $Q_{\mathrm{f}}$ is related to the drag coefficient prefactor $\Phi$ by

$$
\Phi=\frac{4 m_{\mathrm{eff}}}{Q_{\mathrm{f}} A \delta_{\mathrm{n}} \rho_{\mathrm{n}}}
$$

This relation may be used to extract the value of $\Phi$ directly from resonant properties of the oscillator, without precise calibration of driving force or peak velocity. In the laminar regime, it can also be used to infer either force or velocity, provided that the other quantity is known, together with $m_{\text {eff }}$, $A$, and working fluid properties.

The prefactors in the universal scaling law predicted for the oscillators used in this work will be discussed case by case in Sec. III.

\section{Multiple critical velocities in the superfluid}

Here, we comment briefly on the transition to turbulent drag regime observed in the superfluid at very low temperatures corresponding to the ballistic regime. In oscillatory flows under these conditions, a number of experimental studies using vibrating wires [41], grids [26,27], or tuning forks [31,51] reported observation of more than one critical velocity of hydrodynamic origin. Recently, we have presented convincing evidence for three distinct hydrodynamic critical velocities and proposed an explanation linking all the observations of oscillatory flow in zero-temperature limit into a single framework [33].

The first critical velocity, connected mostly to frequency shifts rather than changes in the drag force, is associated with the formation of a number of quantized vortex loops near the surface of the oscillator, possibly forming a thin layer, which affects the coupling to the fluid and thus the hydrodynamic added mass. This first critical velocity is hardly observable in the two-fluid regime above $1 \mathrm{~K}$. The second critical velocity is related to the quantized vorticity propagating into the bulk of the superfluid, either in the form of emitted vortex loops or, eventually, as a turbulent tangle. It is always accompanied by a marked increase in the drag force and usually hysteresis (detectable with amplitude sweeps). We would like to stress that it is this critical velocity which we will be discussing later in relation to the experiments performed in the hydrodynamic regime above $1 \mathrm{~K}$.

For completeness, there is a third critical velocity of hydrodynamic origin, likely associated with the development of larger vortical structures from bundles of polarized quantized vortices. We note that at finite temperature, such polarized vortex bundles or rings have been studied numerically $[52,53]$. The mentioned critical velocity (typically above $1 \mathrm{~ms}^{-1}$ ) might not be relevant in the two-fluid regime at all, as classical features would likely develop in the vortex tangle due to mutual friction even before this mechanism can take effect. 


\section{E. Additional dissipation mechanisms}

In addition to viscous damping, losses due to sound emission through the surrounding fluid may occur, and may be accounted for approximately [54]. In this work, acoustic losses can be safely neglected for the fundamental mode of both tuning forks used and represent perhaps a very small contribution to the damping the first overtone of the custommade fork [55]. Based on our previous studies of acoustic emission by oscillating objects in He II [54,55], acoustic losses are negligible for all other oscillators used in this work. In our experiments, no sign of cavitation and associated losses was detected.

We also note that the above description of viscous dissipation is approximate in the sense that it neglects the steady streaming flow that is known to exist in the vicinity of the oscillating objects and has been recently visualized in He II in highly turbulent flow due to vibrating quartz turning fork [38]. However, the streaming flow has negligible effect on the drag forces measured in laminar viscous flow, as the typical length scale associated with streaming is of order of the size of the oscillator, while the boundary-layer thickness is at least an order of magnitude lower in our experiments. Of course, in turbulent flows, the pressure drag is significantly larger than both the viscous friction and any additional drag due to the streaming.

\section{EXPERIMENTAL DETAILS}

Most of the resonators used in our investigation, the wire, the tuning forks, and the double paddle, were driven by an Agilent A33220 signal generator, and a phase-sensitive Stanford Research SR830 lock-in amplifier was used to measure both the in-phase and out-of-phase components of the induced signals.

The measurements presented here were performed in Prague, mostly in a helium immersion cryostat during a dedicated experimental run for each resonator. The helium bath is brought down to the desired temperature using a rotary pump and a Roots pump and stabilized on the level of few $\mathrm{mK}$ either by manually adjusting the pumping speed or using a temperature controller. The lowest attainable temperature of $1.27 \mathrm{~K}$ allows access to most of the hydrodynamic (two-fluid) regime.

\section{A. Quartz tuning forks}

Quartz tuning forks are piezoelectric oscillators with a calibrated resonant frequency, often used as frequency standards or shear force sensors for scanning optical microscopes [56]. Tuning forks are well-established probes of cryogenic helium flow [30].

The fork is driven by applying an ac voltage $V$ from a function generator to the metallic electrodes deposited on the surface of the quartz. This produces a force proportional to the voltage which sets the two prongs oscillating in antiphase. The distortion of the quartz induces a piezoelectric current $I$ which is proportional to velocity $U$. The relations between force, velocity, voltage, and current are

$$
F=\frac{a_{\mathrm{f}} V}{2} I=a_{\mathrm{f}} U
$$

(a)

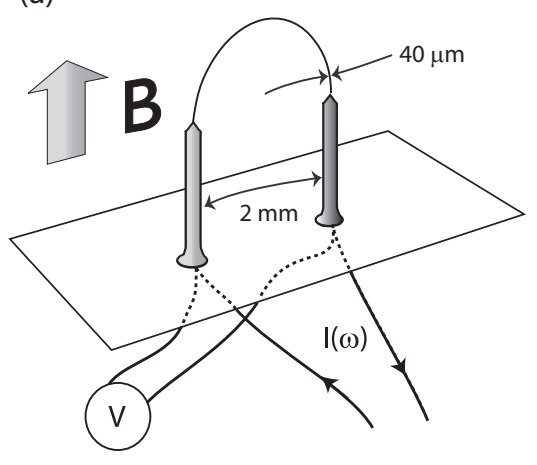

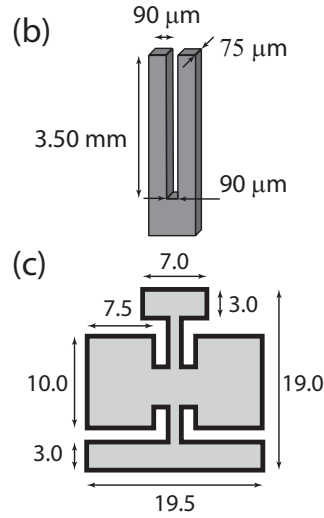

(b)
FIG. 2. Schematic diagrams of the vibrating wire resonator (a), of the quartz tuning fork (b), and the double paddle (c). The dimensions of the double paddle are in millimeters. The wafer thickness is $75 \mu \mathrm{m}$ for the tuning fork and $250 \mu \mathrm{m}$ for the double paddle.

where $a_{\mathrm{f}}$ is the so-called fork constant, which may be obtained through calibration by deflection measurement or selfcalibration in vacuum, in which case it is given as $a_{\mathrm{f}}=$ $\sqrt{4 \pi m_{\mathrm{eff}} \Delta I / V}$, where $m_{\mathrm{eff}}$ is the effective mass of the fork, and $\Delta$ is the measured resonant width [30] at half-height of the (Lorentzian) peak. The effective mass [55] of the tuning fork in vacuum is given by $m_{\text {eff }}=\xi m=T_{\mathrm{f}} W_{\mathrm{f}} L_{\mathrm{f}} \rho_{\mathrm{f}} / 4$, where $\rho_{\mathrm{f}}$ is the density of the fork material (in our case quartz, $\rho_{\mathrm{f}}=2650 \mathrm{kgm}^{-3}$ ), and the dimensions $T_{\mathrm{f}}, W_{\mathrm{f}}, L_{\mathrm{f}}$ stand for the tine thickness (in the direction of motion), width, and length, respectively. The ac current is measured using an IV converter [57] and a SR-830 lock-in amplifier. The standard measurement scheme used here can be found, e.g., in Fig. 1 of Ref. [33].

We have used two different forks in this work. The first is a commercially produced fork of the following dimensions: $L_{\mathrm{f} 1}=2.17 \mathrm{~mm}, T_{\mathrm{f} 1}=210 \mu \mathrm{m}, W_{\mathrm{f} 1}=100 \mu \mathrm{m}$, and the gap between the prongs is $D_{\mathrm{f} 1}=120 \mu \mathrm{m}$. Its surface roughness is $\approx 5 \mu \mathrm{m}$. The second is a custom-made fork with $L_{\mathrm{f} 2}=$ $3.50 \mathrm{~mm}, T_{\mathrm{f} 2}=90 \mu \mathrm{m}, W_{\mathrm{f} 2}=75 \mu \mathrm{m}$ (original wafer thickness), and $D_{\mathrm{f} 2}=90 \mu \mathrm{m}$, with roughness $\approx 1 \mu \mathrm{m}$. A sketch of the fork geometry including the dimensions is shown in Fig. 2. The commercial fork resonates at $32 \mathrm{kHz}$, while with the custom-made fork, we use two different flexural resonant modes: the fundamental resonance at $6.5 \mathrm{kHz}$ and the first overtone at $40.0 \mathrm{kHz}$.

To describe the drag force acting on tuning forks in laminar flow, unfortunately, no analytical solutions of NSE can be obtained. However, significant effort has been invested into studying the dynamical response of rectangular beams immersed in viscous fluids [58,59], resorting to numerical integration to obtain the hydrodynamic response function for rectangular beams of arbitrary aspect ratio. These calculations may thus be applicable to tuning forks. Although we consider $\Phi$ as a parameter to be determined experimentally for each oscillator due to surface roughness effects, we may use the results of Ref. [59] to obtain the approximate dependence $C_{\mathrm{D}}^{\mathrm{n}} \simeq 4.67 / \mathrm{Dn}$ for the custom-made fork (see Appendix A). For the commercial fork, $C_{\mathrm{D}}^{\mathrm{n}} \simeq 5.55 / \mathrm{Dn}$ is obtained in a similar fashion, if its surface roughness is ignored. 


\section{B. Vibrating wire resonator}

Vibrating wire resonators are well-established lowtemperature probes [60]. They consist of a semicircular loop of wire subjected to a vertical magnetic field $B$, as shown in Fig. 2. A loop is used to prevent closely spaced or degenerate modes one may observe on a straight wire.

Traditionally, the vibrating wire is described in the following way. Passing an alternating current $I(\omega)$ through the wire forces it to oscillate due to the Lorentz force $F_{\mathrm{L}}=B D I$. As the wire moves through the magnetic field, it induces a voltage which can be determined using Faraday's law. For a rigid semicircular wire with leg spacing $D$, oscillating at a peak velocity $U_{\mathrm{p}}$, the area bounded by the loop is $A=\pi D^{2} / 8$ and the rate of change of angle to the field is $2 U_{\mathrm{p}} / D$. Therefore, the induced Faraday voltage generated by a semicircular vibrating wire loop is traditionally given by

$$
V=-\frac{d(\boldsymbol{B} \cdot \boldsymbol{A})}{d t} \simeq \frac{\pi}{4} B D U_{\mathrm{p}} .
$$

Here, we argue that the traditional model does not describe the behavior at resonance correctly, in the sense that the energy dissipation at resonance is not equivalent in terms of electrical quantities $\dot{E}_{\mathrm{el}}=1 / 2 \mathrm{VI}$ and within the $1 \mathrm{D}$ mechanical model $\dot{E}_{\text {mech }}=1 / 2 F U_{p}$, as they differ by a factor of $\pi / 4$. This due to the fact that one cannot take the total Lorentz force $F_{\mathrm{L}}$ as the driving force of the resonant mode of the wire, but a projection of this force on the mode shape must be considered. The remaining Lorentz force is driving other resonant modes, as determined by its distribution along the length of the wire, but it does not dissipate any energy, as it is frequency mismatched with respect to those modes (in an off-resonance condition).

A correct definition of the model force may be obtained directly from energy dissipation, as has been done for tuning forks [30]. We use this approach in our proposed model that describes the vibrating wire as a doubly clamped beam. Neglecting for a moment the curvature of the wire (a valid approximation if the wire radius is much smaller than the radius of the loop), the resonant mode shapes may be obtained by solving the Euler-Bernoulli equation. Using the appropriate boundary conditions, one obtains in terms of local velocities

$$
\begin{aligned}
u_{\mathrm{L}}(x) \propto & \left\{\sinh \left(b_{n} x\right)-\sin \left(b_{n} x\right)\right. \\
& \left.-\frac{\left[\cosh \left(b_{n} x\right)-\cos \left(b_{n} x\right)\right]\left[\sinh \left(b_{n} L\right)-\sin \left(b_{n} L\right)\right]}{\cosh \left(b_{n} L\right)-\cos \left(b_{n} L\right)}\right\}
\end{aligned}
$$

for $x \in[0, L]$, where $L$ is the length of the semicircular loop, and $b_{n}=\left(\mu \omega_{n}^{2} / E I\right)^{1 / 4}$, with $\mu$ representing the mass per unit length, $\omega_{n}$ the angular frequency of the $n$th mode, $E$ the Young's modulus, and $I$ the second moment of area of the wire cross section. The resonance frequencies are determined from the equation $\cosh \left(b_{n} L\right) \cos \left(b_{n} L\right)=1$, which has to be solved numerically.

The mode shapes can then be integrated to obtain a modedependent effective mass. For $n=1$, we get $m_{\text {eff }} \approx 0.396 m$. Now taking into account the curvature of the wire to find the changing projected area of the loop on the direction of
$\boldsymbol{B}$ using the obtained mode shape, Eq. (12) will be replaced by $V \approx 0.690 B D U_{\mathrm{p}}$ and the driving force will be given by $F \approx$ $0.690 B D I$. This is the correct projection of the Lorentz force $F_{\mathrm{L}}(x)=B I \sin (\pi x / L)$ on the mode shape of the fundamental resonance, as can be verified by direct integration.

To obtain the drag force in laminar flow, we again neglect the curvature of the loop, approximating each segment along the length of the wire as a smooth cylinder oscillating with a local velocity amplitude $u_{\mathrm{L}}(x)$. The drag force per unit length acting on such a cylinder is given, e.g., in Ref. [47]. Following the procedure outlined in Sec. II C, for the fundamental mode, the drag coefficient is given as $C_{\mathrm{D}}^{\mathrm{n}}=4 \pi \xi / \mathrm{Dn} \approx 4.98 / \mathrm{Dn}$.

The vibrating wire resonator used in this study consists of a semicircular loop of superconducting NbTi wire with a leg spacing of $D=2 \mathrm{~mm}$ and a diameter of $2 R=40 \mu \mathrm{m}$. The wire was mounted in a brass cell submerged in the bulk superfluid and mounted between a pair of $\mathrm{NdFeB}$ permanent magnets in a magnetic field of $(170 \pm 10) \mathrm{mT}$ at room temperature. We estimate that the field is reduced by approximately $23 \%$ at low temperatures [61] due to spin reorientation occurring in $\mathrm{NdFeB}$ at $135 \mathrm{~K}$. Given the uncertainty of the magnetic field, we have used Eq. (10) to obtain a self-calibration of the force driving the vibrating wire.

\section{Double paddle}

Recent studies [36,37] have shown that double-paddle oscillators (DPOs) may serve as promising probes to study superfluid hydrodynamics. They have demonstrated highquality factors in vacuum compared to other mechanical resonators since any vibrational losses through their base are heavily suppressed.

Here, we reanalyze the results obtained with the silicon DPO etched from a 0.25 -mm-thick $\langle 110\rangle$ wafer used by Zemma and Luzuriaga [36], sketched in Fig. 2. The two larger wings are approximately $10 \mathrm{~mm} \times 7.5 \mathrm{~mm}$ and the smaller upper paddle is $7 \mathrm{~mm} \times 3 \mathrm{~mm}$. The DPO was driven magnetically by attaching a small magnet located between the wings in the oscillator stem; its displacement was detected capacitively. In order to generate the oscillatory motion, an ac current was applied to a small superconducting coil fixed to the support frame.

The complex geometry of the DPO precludes any analytical solutions of NSE, and we are not aware of any numerical studies detailing the laminar drag experienced by a submerged DPO.

\section{Torsionally oscillating disk}

The torsional oscillator consists of a $0.05-\mathrm{mm}$ tungsten wire, $32 \mathrm{~cm}$ long, with a borosilicate glass disk fixed to the wire at its midpoint using a thin $0.8-\mathrm{mm}$ brass capillary and Stycast $2850 \mathrm{GT}$. The disk is $1 \mathrm{~mm}$ thick with a diameter of $40 \mathrm{~mm}$; a schematic diagram is shown in Fig. 3. When the wire is under tension, the disk is positioned approximately midway between the two copper-coated, polished FR-2 plates placed $10 \mathrm{~mm}$ apart (both disk sides are approximately $4.5 \mathrm{~mm}$ away from the FR-2 plate facing them). The deflection and angular velocity of the disk are determined from recorded video sequences as detailed in Appendix B. 


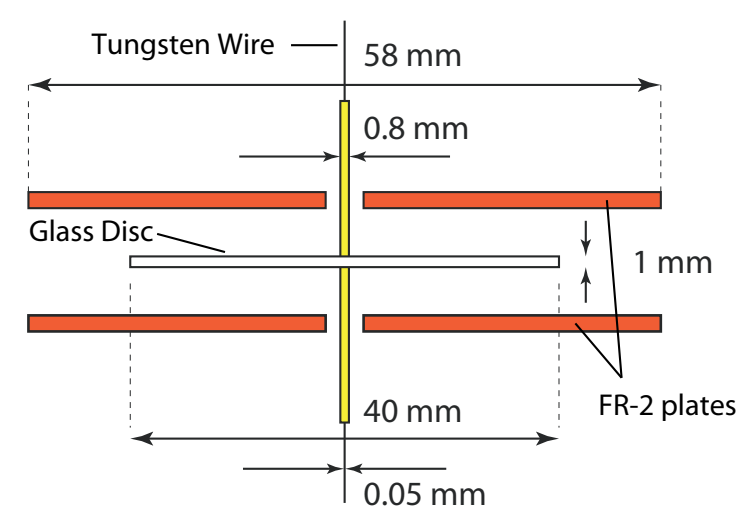

FIG. 3. Schematic diagram of the torsionally oscillating disk.

To facilitate comparison with other oscillators, we define a drag coefficient for a thin disk torsionally oscillating in a viscous fluid of density $\rho_{\mathrm{n}}$ as

$$
\mathrm{C}_{\mathrm{D}}^{\mathrm{n}}=\frac{2 M_{\mathrm{F}}}{A \rho_{\mathrm{n}} \Omega_{0}^{2} R^{3}},
$$

where $M_{\mathrm{F}}$ is the moment of friction forces, $R$ is the disk's radius, $A=\pi R^{2}$ is the surface area of one side of the disk, $\Omega_{0}$ is the amplitude of the angular velocity, and $\omega$ is the angular frequency of oscillation. For a rationale of this definition, and for the derivation of the Donnelly number dependence, we refer the reader to Appendix C. In laminar flow, the drag coefficient due to the normal component can be expressed in terms of the Donnelly number as $C_{D}^{n}=2 / D n$.

\section{EXPERIMENTAL RESULTS AND ANALYSIS}

In this section we present our drag force measurements using the resonators introduced above and compare the results against the proposed universal scaling law.

\section{A. Tuning forks}

The custom-made tuning forks used in our measurements are fully described and characterized in Ref. [33]. By performing frequency sweeps in vacuum at low temperature, the experimental fork constant is estimated to be $a_{\mathrm{f}}=3.665 \times$ $10^{-7} \mathrm{C} / \mathrm{m}$ and $a_{\mathrm{f}}^{\mathrm{o}}=1.409 \times 10^{-6} \mathrm{C} / \mathrm{m}$ for the fundamental mode and first overtone, respectively. We estimate that the fork constant has an uncertainty of $10 \%$ since it was shown that the optically measured prong velocity can be $10 \%$ lower [62] than that determined from the electromechanical model described in Sec. III A. The details of the commercial fork are given in Ref. [50], where it is labeled "L2."

Figure 4 shows typical results for the drag offered by He II to driven oscillations of the quartz tuning fork and compares them to the numerical results of Ref. [59]. In the left of Fig. 4, we plot the classical drag coefficient as a function of the peak velocity at various temperatures. As expected, the tuning forks exhibit linear damping at low velocities at all temperatures. Upon increasing the velocity, the drag coefficient tends to a temperature-independent constant value of order unity $\left(\mathrm{C}_{\mathrm{D}} \approx 0.6\right)$ as one would expect for fully coupled normal and superfluid components. The flow due to the fork then behaves as a single classical-like fluid in the turbulent drag regime. On decreasing temperature, the drag coefficient drops appreciably over the range of low and intermediate velocities as the density of the normal fluid component decreases. This is in agreement with previous analysis [50].

To characterize the flow of the normal component, we plot the normal fluid drag coefficient as a function of the Donnelly number in the right of Fig. 4. At low Donnelly numbers, the data collapse to a single dependence for each fork, before deviating at some critical value. Note that despite the difference in the velocity profile and the viscous penetration depth, the same prefactor $\Phi$ in Eq. (6) is obtained for the two resonant modes of the custom-made fork, supporting the validity of the derived scaling law. This is due to the fact that both modes have the same flow enhancement factor $\alpha$ determined by the rectangular cross section of the prong and practically the same effective mass $m_{\text {eff }}=\xi m+m_{\mathrm{HD}}$ with $\xi=\frac{1}{4}$ (see Appendix A of Ref. [55]). Furthermore, the obtained prefactor $\Phi$ agrees almost perfectly ( $\approx 2 \%$ deviation) with Ref. [59] (see calculation in Appendix A). Careful inspection also reveals differences in the onset of nonlinear drag for the lowest two temperatures (this will be further analyzed in Sec. IV E). The commercial fork shows the same universal scaling, but the obtained prefactor is $1.4 \times$ higher than the numerical result. This is likely due to surface roughness effects. Comparison to oscillations in classical liquid helium and helium gas is shown on the commercial fork data, where $\mathrm{Dn} \equiv \mathrm{Re}_{\delta}$ is used, highlighting the same form of the scaling law in both classical and quantum fluids. As the commercial tuning fork is hydrodynamically rough, a unique dependence $C_{\mathrm{D}}^{\mathrm{n}}(\mathrm{Dn})$ is expected in classical fluids as well as wherever the superfluid component does not contribute to the drag force appreciably. This is illustrated in the lower right panel of Fig. 4, as the data obtained in $\mathrm{He} \mathrm{I}, \mathrm{He}$ gas, and at $T=2.16 \mathrm{~K}$ agree quite well over the entire range of Dn. Departures from this dependence mark drag forces originating from the superfluid component, or arising in either component due to their coupling by mutual friction.

\section{B. Vibrating wire resonator}

The resonant response of the vibrating wire resonator is obtained by measuring the voltage in phase with the driving current, as a function of frequency. In accord with previous works $[41,42,60]$, for small drive levels, the frequency response is of Lorentzian form. Upon increasing the drive level, the Lorentzian shape becomes distorted and the resonant frequency decreases. The flattening of the peak indicates the onset of nonlinear drag forces typically associated with turbulent instabilities in the generated oscillatory flow.

The classical drag coefficient as a function of velocity for the vibrating wire is plotted in the left of Fig. 5. In order to collapse the contribution of the normal fluid component to the drag forces acting on the wire to a single dependence, we again plot the drag coefficient for the normal component as a function of the Donnelly number [see Eq. (6)] in the right of Fig. 5. Universal scaling with the Donnelly number is observed for the wire, up to critical value, which is now, however, temperature dependent, in striking difference with the custom-made tuning fork. We also note that the prefactor 


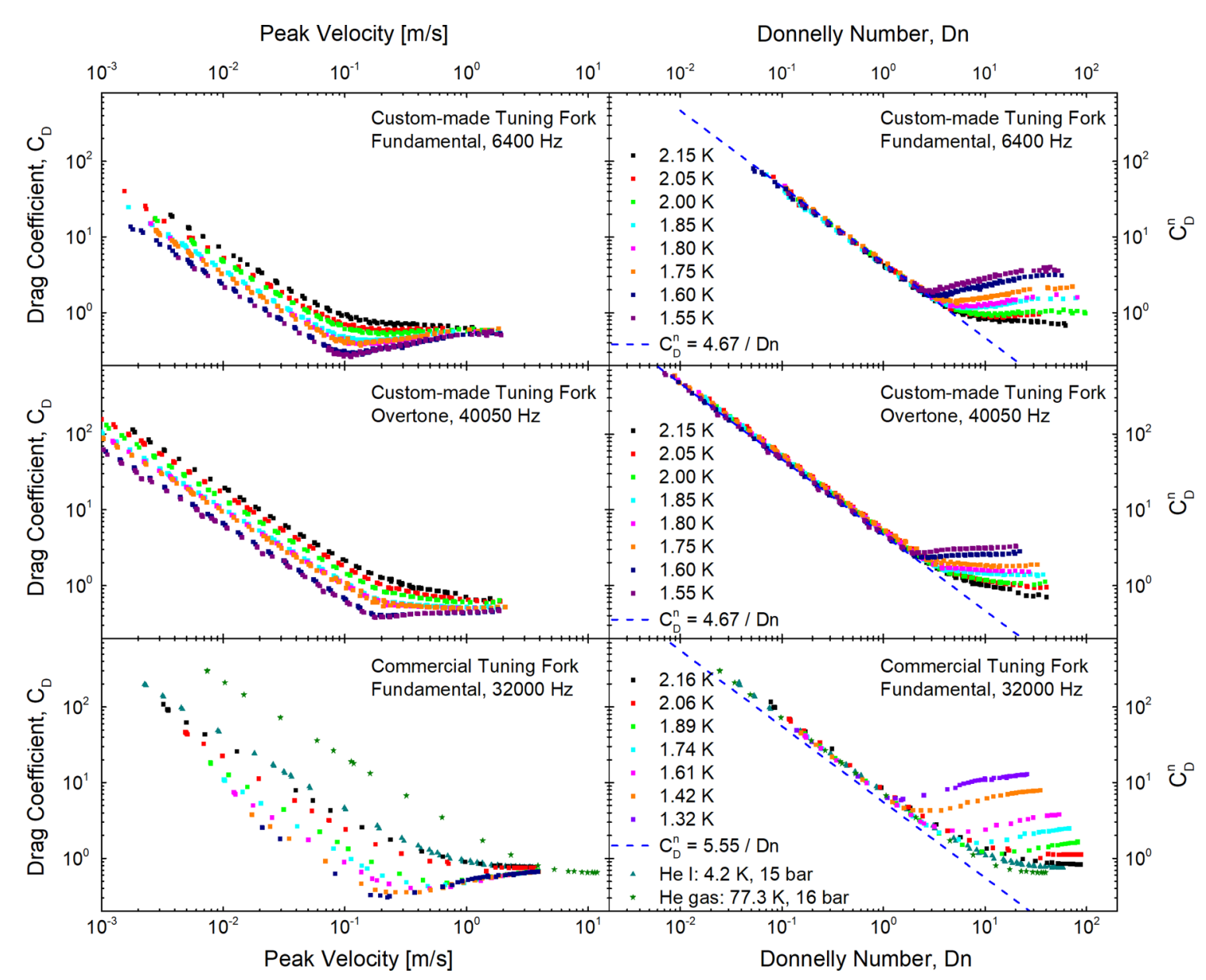

FIG. 4. Left: drag coefficient as function of velocity for the quartz tuning forks. Right: the corresponding normal fluid drag coefficient as a function of the Donnelly number. Note that (i) the same prefactor for the laminar scaling is displayed for the fundamental mode and overtone of the custom-made tuning fork, in near perfect agreement with the calculation described in the text and that (ii) for commercial fork, the same scaling is observed in classical (He I, He gas) and quantum (He II) fluids. A slight disagreement in the prefactor with respect to the numerical calculations is observed: the experimental data can be recovered by applying a multiplicative factor of 1.4 , which we associate with the surface roughness of the commercial fork.

for the laminar drag is by $10 \%$ to $15 \%$ smaller than calculated. This is most likely due to the uncertainty in the wire radius and hence in its effective mass, which enters Eq. (10) that was used to obtain the driving force from resonant properties. While the 2-mm wire loop was located in a cylindrical cavity of diameter $4 \mathrm{~mm}$, we do not expect a significant effect of the container walls on the measured drag, as the viscous penetration depth $\delta_{\mathrm{n}}$ is of order $1 \mu \mathrm{m}$.

\section{Double paddle}

We now apply the same analysis to results obtained using a silicon DPO by Zemma and Luzuriaga [36]. Specifically, we

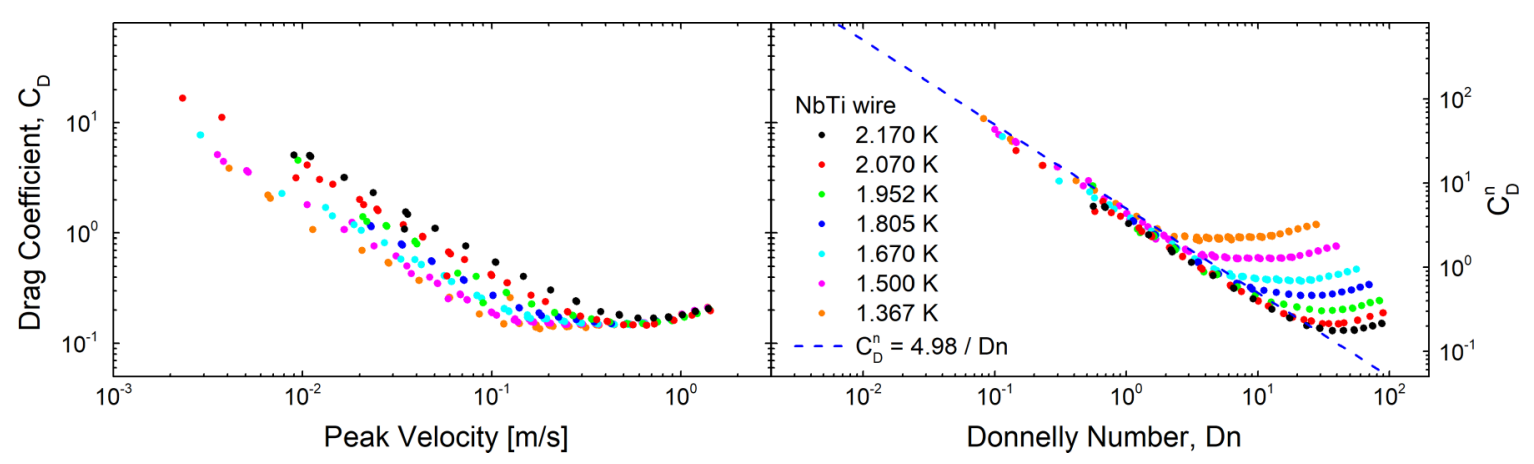

FIG. 5. Drag coefficients as functions of the peak velocity or Donnelly number obtained for the vibrating wire. 


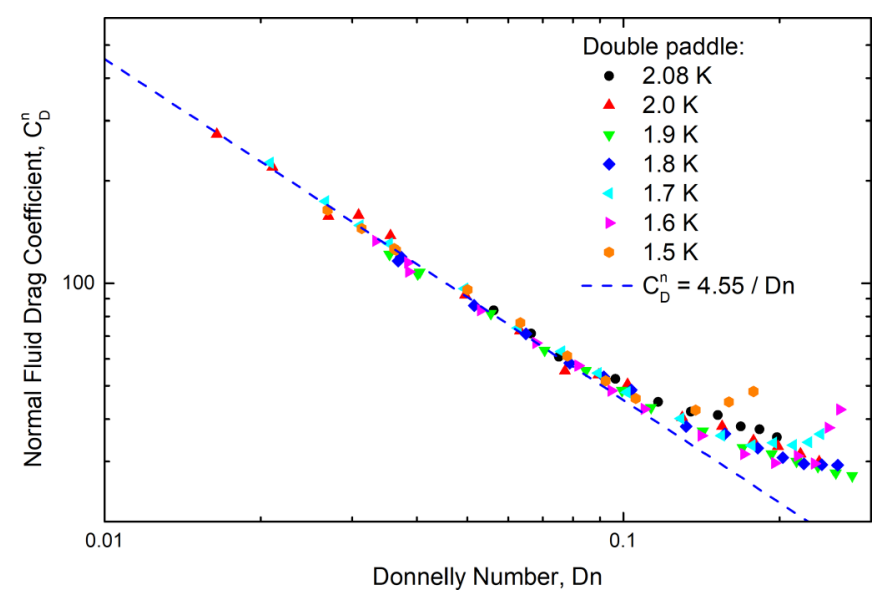

FIG. 6. Normal fluid drag coefficient as a function of the Donnelly number calculated for the silicon double paddle of Zemma and Luzuriaga [36].

analyze the symmetric torsion mode data [35]. In vacuum at $\approx 4.2 \mathrm{~K}$, the resonant frequency of the paddle is $520 \mathrm{~Hz}$, in liquid helium at $4.2 \mathrm{~K}$ it is $358 \mathrm{~Hz}$. The viscous penetration depth is $\approx 3 \mu \mathrm{m}$. Since the lateral characteristic length scale of the paddle is $D \simeq 7 \mathrm{~mm}$, and the thickness is $250 \mu \mathrm{m}$, the paddle is operating in the high-Stokes-number limit, justifying our analysis.

In Fig. 6, we present the normal fluid drag coefficient plotted against the Donnelly number. The viscous drag force again collapses to a single dependence within an uncertainty of $\pm 15 \%$, demonstrating that the paddle is indeed in the highStokes-number limit. The drag force offered by the normal fluid is again described by the same universal scaling law, even for an oscillator of significantly different shape than a wire or tuning fork, in this case following approximately $\mathrm{C}_{\mathrm{d}}^{\mathrm{n}}=4.55 / \mathrm{Dn}$. To the best of our knowledge, no theoretical or computational works exist that would allow a quantitative comparison of the prefactor.

\section{Torsionally oscillating disk}

The torsionally oscillating disk differs from the previous oscillators in three fundamental ways. First, as the disk oscillates around its axis, it does not displace any fluid, hence, there is no potential flow outside the boundary layer. Second, in this case we are not able to perform measurements in a steady state and we have to deal with slowly decaying oscillations of the disk and of the flow due to its motion. Third, we cannot directly measure the drag force and have to infer the damping from the decaying amplitude of oscillation. Despite these important differences, we seek to analyze the flow in a manner similar to the above oscillators.

First, we have established that the intrinsic damping of the disk is negligible compared to that due to the surrounding helium. This was done by measurements in vacuum at room temperature and $78 \mathrm{~K}$, and already at $78 \mathrm{~K}$ the intrinsic damping was far below any measured in superfluid helium. We note, however, that the entire tungsten filament had to be submerged in helium in order to ensure that its temperature is sufficiently low, as it was connected to the driving mechanism

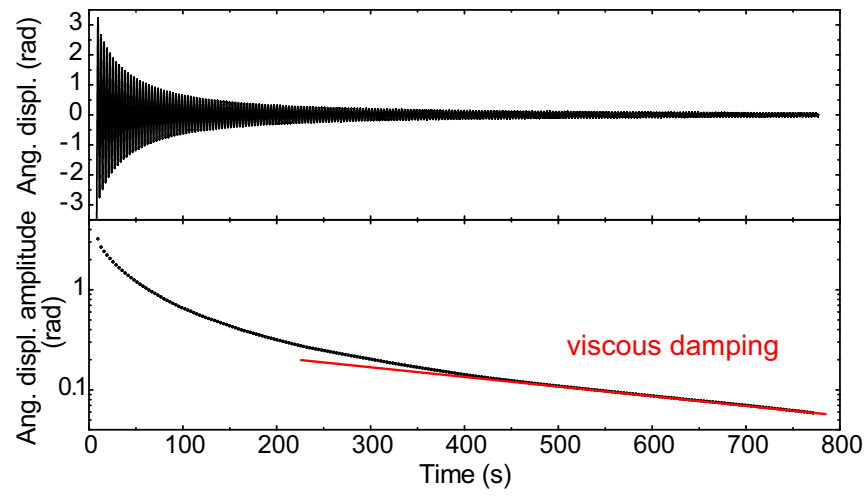

FIG. 7. Typical measurement of angular displacement of the torsionally oscillating disk in He II as a function of time. (Top) The signal extrema were evaluated to obtain the angular displacement amplitude $\phi_{0}$. The logarithmic plot (bottom) clearly shows two distinct regions: exponential (viscous) decay due to laminar flow of the normal component for $t \gtrsim 500 \mathrm{~s}$ and a faster nonlinear decay at earlier times, related to turbulent drag. The position of the disk oscillating with a period of $T \simeq 3.17 \mathrm{~s}$ is sampled at $240 \mathrm{~Hz}$ (see Appendix B). The turbulent decay is typically observed on timescales of order $100 \mathrm{~s}$, whereas decays of coflow or counterflow turbulence in He II typically in a few seconds.

at the top flange by a thin-walled stainless steel tube with no special regards for thermal isolation.

As the moment of frictional forces $M_{\mathrm{F}}$ cannot be obtained directly from the experiment, we have to infer the drag coefficient from other measurable quantities, such as the extremal displacements of the disk during its damped oscillations as shown in Fig. 7. If the series of extremal angular displacements occurring at times $t_{n}$ is labeled $\varphi_{n}$ (interleaving maxima and minima in chronological order), the logarithmic decrements of the amplitude of oscillation $\alpha_{n}$ are determined as $\alpha_{n}=\ln \left(\varphi_{n-1}\right)-\ln \left(\varphi_{n+1}\right)$ and the immediate angular frequency of oscillation is $\omega_{n}=2 \pi /\left(t_{n+1}-t_{n-1}\right)$. This leads to an alternative definition of the drag coefficient:

$$
\mathrm{C}_{\mathrm{D}}^{\mathrm{n}}=\frac{2 I \alpha}{\pi A \rho_{\mathrm{n}} R^{3} \varphi_{0}} \simeq \frac{\rho_{\mathrm{d}} h_{\mathrm{d}} \alpha}{\pi \rho_{\mathrm{n}} R \varphi_{0}},
$$

where $\varphi_{0}$ denotes the immediate angular displacement amplitude, and $I=I_{0}+I_{\mathrm{HD}}$ stands for the effective moment of inertia consisting of the moment of inertia of the disk itself $I_{0}$, and of its hydrodynamic enhancement $I_{\mathrm{HD}}$. If $I_{\mathrm{HD}} \ll I_{0}$, the simplified expression on the right-hand side of Eq. (15) holds, where $\rho_{\mathrm{d}}$ is the density of the disk material and $h_{\mathrm{d}}$ the height (thickness) of the disk. The derivation can be found in Appendix C.

We plot the drag coefficient $\mathrm{C}_{\mathrm{D}}^{\mathrm{n}}$ measured at various temperatures against the Donnelly number Dn $=\rho_{\mathrm{n}} \delta_{\mathrm{n}} R \omega \varphi_{0} / \eta$ in Fig. 8. At small values of $\mathrm{Dn}$, the data collapse to a single dependence illustrating the universal behavior. As the disk is hydrodynamically smooth, we do not expect the turbulent instability to occur at a well-defined critical value of Dn, but we may still be able to distinguish between a classical instability in the normal component and the onset of superfluid turbulence by considering the dependence of the nonlinear drag on the densities $\rho_{\mathrm{n}}$ and $\rho_{\mathrm{s}}$. 


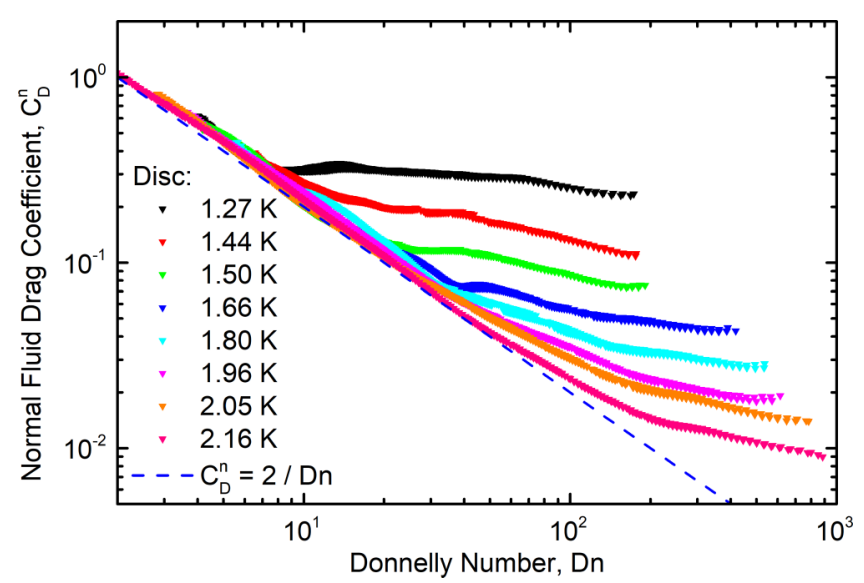

FIG. 8. Normal fluid drag coefficient as a function of the Donnelly number for the torsionally oscillating disk at the various indicated temperatures. The dashed blue line is the predicted dependence for viscous drag $C_{D}^{n}=2 / D n$. Prior to the calculation of $C_{D}^{n}$, the logarithmic decrements $\alpha_{n}$ have been smoothed by a 50-point weighted adjacent averaging filter and checked against the original data, showing minimal deviation.

\section{E. Analysis of instabilities}

While the drag coefficients shown in the previous sections contain, in principle, all necessary information about the flow properties, it is useful to examine the transition to nonlinear drag in more detail. In particular, we are interested in determining which type of instability occurs upon increasing oscillation amplitude first: a classical instability of the normal component or the multiplication of remnant quantized vortices in the superfluid component?

To tackle this issue, we need to analyze the first departures from laminar drag, hence, we withdraw from the measured drag force the part that is linear with velocity, keeping only the nonlinear contribution. Such a quantity needs to be normalized and plotted against parameters relevant to either component in order to deduce the nature of the first detected instability. It seems particularly advantageous to use the quantity $1-\Phi /\left(C_{D}^{n} D n\right)$ in a plot against Dn to describe the action of the normal component and, analogically, $1-\phi /\left(\mathrm{C}_{\mathrm{D}}^{\mathrm{s}} \hat{\mathrm{U}}\right)$ against $\hat{U}$ for the superfluid component [see Eq. (7)]. These definitions guarantee that the result is always close to zero in laminar flow, and approaches one as the nonlinear drag starts
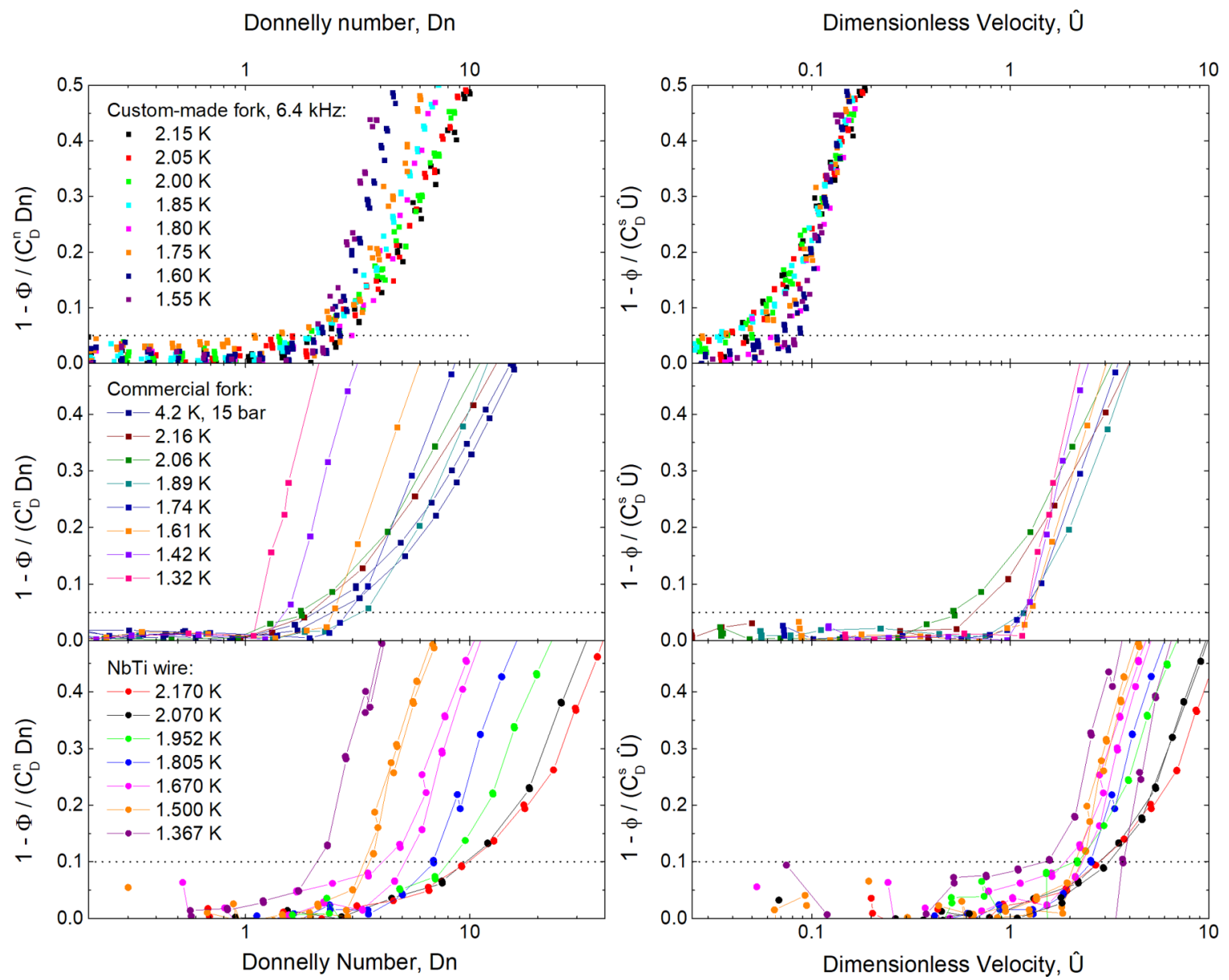

FIG. 9. Turbulent instability analysis for both tuning forks and the vibrating wire resonator. Left: nonlinear drag normalized using normal component properties versus Donnelly number. Right: nonlinear drag normalized using superfluid component properties versus nondimensional velocity $\hat{U}$. We note that the quantities on the ordinate axes are equivalent, as both represent the ratio of the nonlinear drag to the total drag experienced by the oscillator. 

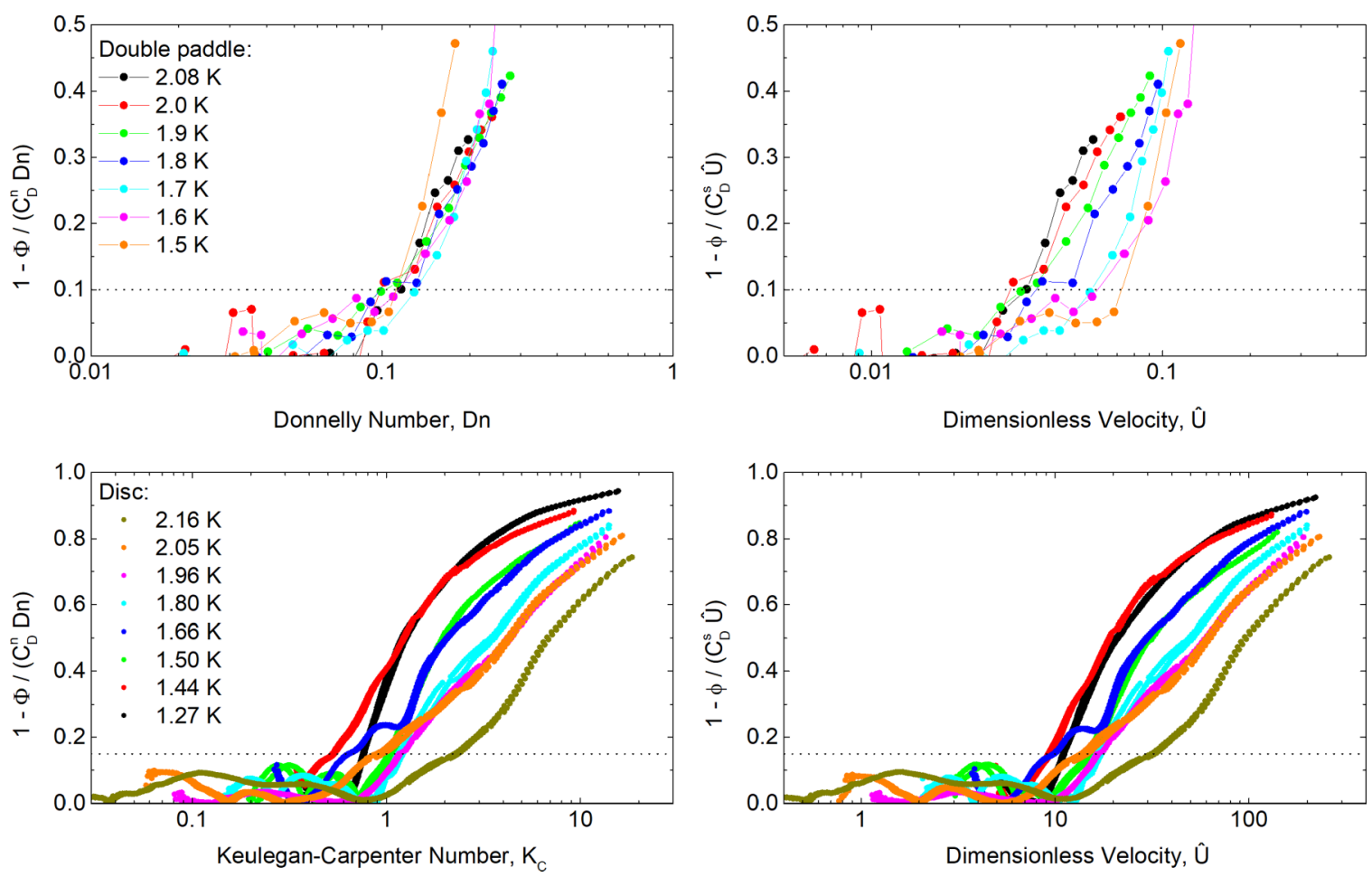

FIG. 10. Turbulent instability analysis for the double paddle and the disk. Left: nonlinear drag normalized using normal component properties versus the Donnelly number or Keulegan-Carpenter number, as applicable. Right: nonlinear drag normalized using superfluid component properties versus nondimensional velocity $\hat{U}$.

to dominate. For the oscillating disk, $\mathrm{K}_{\mathrm{C}}$ is used instead of Dn, in agreement with the theory in Sec. II B.

Such plots are shown in Fig. 9 for the two tuning forks and the vibrating wire resonator, with each oscillator displaying different behavior. We consider the instability occurring at a given departure from the linear drag, which must be above the experimental noise level in the data acquired in laminar flow. For the tuning forks, we use a 5\% departure criterion, for the wire, $10 \%$ seems more appropriate. To understand the results, it is useful to consider two aspects: (i) the magnitude and relative spread of critical values of either Dn or $\hat{U}$ when crossing the given threshold, (ii) the rate at which the nonlinear drag sets in.

In the top two panels of Fig. 9, the custom-made fork shows a notably lower spread in Dn than in $\hat{U}$, signifying that Dn is likely to be the correct parameter governing the (classical) instability in a larger part of the range of temperatures investigated. On the other hand, the vibrating wire resonator (bottom two panels) displays a rather well-defined critical value of $\hat{U}$, while showing significant spread in Dn (except for the two highest temperatures, for which the critical values of Dn coincide), giving evidence of a Donnelly-Glaberson type of instability in the superfluid component. The commercial tuning fork (middle panels) shows a clear crossover: at temperatures below $2.0 \mathrm{~K}$ the instability is governed by $\hat{U}$, while at higher temperatures it is determined by $\mathrm{Dn}$. It is interesting to note that whenever the instability is determined by $\hat{U}$, the onset of nonlinear drag is notably sharper. A crossover between a classical and quantum instability might be present in the other two oscillators as well, but is not as pronounced as with the commercial tuning fork.
The presented interpretation is further supported by the observed critical values of the governing parameters. For the commercial fork, the critical dimensionless velocity $\hat{U}_{\mathrm{C}} \approx$ 1.2 , and for the vibrating wire resonator values between 1.5 and 3 are found. However, the custom-made fork has only $\hat{U} \approx 0.1$ when the nonlinear drag sets in. Hence, the Donnelly-Glaberson instability is very unlikely to occur, and is preceded by the classical instability near $\mathrm{Dn}_{C}=2.5$. Furthermore, the (minimum) critical value of Dn characterizing the classical instability can be obtained from measurements in classical fluids, such as He I or He gas, or from experiments very close to $T_{\lambda}$ where the drag offered from the very low density superfluid component can be neglected. Hence, for the commercial fork we obtain $\mathrm{Dn}_{\mathrm{C}} \approx 2.5$ and for the wire we get $\mathrm{Dn}_{\mathrm{C}}^{\mathrm{m}}$ in $\approx 9$ from the data at 2.07 and $2.17 \mathrm{~K}$. The lower value of $\mathrm{Dn}_{\mathrm{C}}$ obtained for the forks is likely related to velocity enhancement in flow past its sharp corners.

In Fig. 10 we analyze the data from the DPO and the torsionally oscillating disk in a similar manner. For the DPO we find a classical instability in the entire temperature range, characterized by a critical value of the Donnelly number $\mathrm{Dn}_{\mathrm{C}} \approx 0.1$, with the rather low value again related to flow enhancement. Indeed, in the symmetric torsion mode of the $\mathrm{DPO}$, the displaced fluid needs to move significantly faster than the oscillator itself to flow from one side of the wings to the other and back during one period of oscillation.

For the disk, the situation is more complex and fundamentally different from the oscillators just discussed, for several reasons. In analyzing the data, we need to bear in mind that contrary to the other oscillators, the disk is hydrodynamically smooth, and hence the instability should be governed by 
the Keulegan-Carpenter number $\mathrm{K}_{\mathrm{C}}$. Unfortunately, $\mathrm{K}_{\mathrm{C}}$ scales with the fluid properties in a very similar fashion to the dimensionless velocity $\hat{U}$, making our situation complicated. The spread of critical values of both parameters is very similar, and the numerical critical values are in both cases acceptable. For comparison, if the data in the bottom left panel in Fig. 10 were plotted against Dn, the critical values would show a very large spread between 8 and 100 (see Fig. 8). However, since the data taken at $2.16 \mathrm{~K}$ (where fluid properties ought to be dominated by the normal component) differ significantly from the all the other series, we are led to believe that except for this highest temperature, the instability has origins in the superfluid component.

Furthermore, since the disk is set into motion at a high amplitude and left to oscillate, we are dealing with a decaying turbulent flow; this has implications for our interpretation, if hysteresis exists at the turbulent transition. Here, we emphasize that temporal decays of quantum turbulence usually observed in both coflow and counterflow geometries are typically much faster than the observed timescale of the decay of torsional oscillations. We thus believe that the intensity of quantum turbulence is, at all times, near its steady-state value determined by the immediate amplitude of oscillations of the disk. Nevertheless, the observed critical values do not signify the first instability occurring in a laminar flow as with the other oscillators, but rather a minimum requirement, a necessary condition for preexisting turbulence to survive, which might generally depend on details of the turbulent flow. Such a requirement seems to be given by $10<\hat{U}_{\mathrm{C}}<20$ for all the investigated temperatures except for $2.16 \mathrm{~K}$, where a higher critical velocity is observed.

To the best of our knowledge, there are two possible reasons for this behavior. First, it is likely that most of the nonlinear drag observed at $2.16 \mathrm{~K}$ above $\hat{U}_{\mathrm{C}} \approx 30$ is in fact due to the normal component which behaves independently from the superfluid and undergoes its own instability at $\mathrm{K}_{\mathrm{C}} \approx$ 2 , corresponding to $\hat{U}_{\mathrm{C}} \approx 30$. The nonlinear drag from the superfluid component (still present) might then be below our resolution. The second possibility is that at $2.16 \mathrm{~K}$, the significantly enhanced damping of the motion of quantized vortices in $\mathrm{He}$ II is responsible for the dissipation of any existing quantum turbulence (the dissipative part of mutual friction force grows steeply with temperature close below the superfluid transition [11]). This seems plausible especially in a situation with no large-scale flow of the superfluid component to provide a supply of energy, as in our case the superfluid is not displaced by the motion of the torsionally oscillating disk.

\section{DISCUSSION}

Let us summarize the experimental results on the two-fluid He II flows due to several types of mechanical oscillators. In all of them, the normal fluid flow (as well as the corresponding flow of classical viscous normal He I) is characterized by high Stokes number, and for low velocities it is laminar. In this limit, the superflow is either potential or, in the case of the oscillating disk, the superfluid component remains stationary in the laboratory frame of reference (barring a low density of pinned remnant vortices [63]). We therefore have two (almost) independent velocity fields, and flows of the normal and superfluid components can be treated independently. It is therefore natural to treat the normal fluid as classical viscous fluid and it is not surprising that the drag coefficient $C_{D}^{n}$ due to the normal fluid displays universal scaling in terms of the Donnelly number Dn. Assuming that the flow of the superfluid component remains potential, upon increasing the Donnelly number the universal scaling holds and, for hydrodynamically rough bodies, describes instabilities in the normal flow leading to gradual transition from laminar to turbulent drag regime in the normal fluid flow. The normal fluid flow is no longer laminar and the overall He II flow can be characterized as quantum turbulence in the sense of a vortical flow occurring in a quantum fluid, despite that there are almost no quantized vortices present.

In some of the investigated oscillatory two-fluid He II flows, the opposite situation appears in that the critical velocity associated with the Donnelly-Glaberson instability in the superfluid component occurs first, before the instability in the normal fluid flow develops. This situation is not new in superfluid hydrodynamics. Indeed, in typical experiments with rotating superfluid ${ }^{3} \mathrm{He}-\mathrm{B}$ the thick normal component virtually does not move in the laboratory frame of reference [64]. Still, below about half of the critical temperature $T_{\mathrm{c}}$ the dissipative mutual friction coefficient falls below unity [65] and a tangle of quantized vortices, superfluid turbulence, can exist in the soup of a thick stationary normal fluid.

In He II experiments with oscillators described above, the situation is different in that the quantized vorticity coexists with the laminar boundary layer flow of the normal component. In ${ }^{4} \mathrm{He}$, this situation is reported and analyzed in this work and is best illustrated for the case of He II flow due to the vibrating wire (see Fig. 9).

Now, as the Donnelly-Glaberson instability occurs upon reaching a critical velocity, but the instability in the normal fluid flow is governed upon reaching a critical Donnelly number, a crossover is possible, thanks to the steep temperature dependence of the kinematic viscosity of the normal fluid. In other words, in the particular example of He II flow due to the commercial tuning fork (see again Fig. 9) at high temperatures, close to the superfluid transition temperature $T_{\lambda}$, the classical instability in the normal fluid is reached first, while at low temperatures the situation is reversed in favor of the Donnelly-Glaberson instability. The existence of this crossover is, remarkably, reported here despite the immense effort in investigating oscillatory flows in He II, especially during the last two decades.

Either instability eventually serves as a trigger for the other one, mediated by the mutual friction force or fluctuating pressure forces, until in the limit of high velocities, both fluids are tightly coupled in the vicinity of the oscillator and He II behaves as a single-component quasiclassical fluid.

\section{CONCLUSIONS}

We have performed systematic measurements of highStokes-number flows of He II due to oscillatory motion of selected oscillators: vibrating wire resonator, tuning forks, double paddle, and torsionally oscillating disk, over a broad temperature range where our working fluid, He II, displays 
the two-fluid behavior. We have shown that in this class of flows the origin of any instability in the normal or superfluid component can be determined by complex drag force analysis, based on which one can separate the drag offered to these oscillators by the normal and superfluid components of He II. For low velocities, we observe universal viscous drag scaling in terms of the suitably defined drag coefficient $C_{D}^{n}$ and the normal fluid boundary-layer-based Reynolds number which we call the Donnelly number Dn.

The superfluid component does not contribute to the drag until an instability associated with extrinsic production of quantized vorticity occurs, governed by the dimensionless velocity $\hat{U}=U / \sqrt{\kappa \omega}$. The underlying physics involves Donnelly-Glaberson instability, i.e., self-reconnections of quantized vortices upon reaching a critical velocity. Until then, the flow of the superfluid component is either potential (excepting pinned remnant vortices) with the superfluid component playing a role of a physical vacuum, renormalizing the hydrodynamic effective mass of the oscillators, or (in the case of the torsionally oscillating disk) the superfluid component remains stationary in the laboratory frame of reference.

Which instability (i.e., classical hydrodynamic instability of laminar flow of the normal component or DonnellyGlaberson instability in the superfluid component) occurs first depends both on the geometry of the oscillator and temperature. We observe a crossover between these instabilities, thanks to the steep temperature dependence of the kinematic viscosity of the normal fluid. Upon increasing oscillation amplitude, either instability can live on its own until eventually it serves as a trigger for the other one, mediated by the mutual friction force or by pressure forces. At high velocities, both fluids are tightly coupled in the vicinity of the oscillator and He II behaves as a single-component quasiclassical fluid.

We believe that the described approach, i.e., treating the flows of normal and superfluid components of He II independently, can be extended and applied to different twofluid He II flows, such as different types of coflows (where the normal and superfluid components are forced together) but perhaps also to the more general case of counterflows (where a nonzero difference of mean velocities of normal and superfluid components exists), in particular to special cases known as thermal counterflow and pure superflow. One can find known features of these flows, such as temperature dependence of the onset of quantum turbulence at various geometries, which provide hints that this approach will most likely be useful, however, dedicated detailed experiments are needed to fully resolve the long-standing puzzles of superfluid hydrodynamics such as the existence of experimentally observed [66] turbulent states TI, TII, and TIII in thermal counterflow and pure superflow. We believe that our results will stimulate further research of the fascinating topic of superfluid hydrodynamics and quantum turbulence.

All data used in this paper are available in Ref. [67], including descriptions of the data sets.

\section{ACKNOWLEDGMENTS}

We thank E. Zemma and J. Luzuriaga for providing the data of Ref. [36] for further analysis, L. Doležal for skillful manufacturing of various cryogenic parts of the experiments, and E. Collin, V. S. L'vov, M. Rotter, and K. R. Sreenivasan for fruitful discussions. This research is funded by the Czech Science Foundation under Project No. GAČR 17-03572S and UK EPSRC Grants No. EP/P022197/1 and No. EP/L000016/1. M.J.J. acknowledges personal support from Vakuum Praha spol. s r.o. V.T. would like to acknowledge the support of the European Microkelvin Platform EMP.

\section{APPENDIX A: DERIVATION OF TUNING FORK DRAG COEFFICIENT}

In Ref. [59], numerical calculations are used to evaluate the inertial and drag forces per unit length acting on uniformly oscillating rectangular cylinders. The cylinders are assumed infinite, with the same cross section everywhere. The drag force amplitude per unit length is expressed in Eq. (2) of Ref. [59] as

$$
f_{1} d l=\frac{\pi}{4} \rho \omega^{2} X^{2} W_{\mathrm{cyl}} \Gamma(\omega),
$$

where $\rho$ is the fluid density, $\omega$ the angular frequency of oscillation, $X$ a dominant length scale which corresponds to the larger dimension of the beam cross section, $W_{\text {cyl }}$ is the displacement amplitude, and $\Gamma(\omega)$ is a complex-valued hydrodynamic response function. This function is then evaluated numerically for cylinders of selected aspect ratios at selected values of a modified Stokes number $\beta_{d}$, where $\beta_{d}=\omega d^{2} / \nu$, and $d=X / 2$. The real and imaginary parts of $\Gamma(\omega)$ correspond to inertial and dissipative forces, respectively; we will thus need to evaluate only the imaginary part $\operatorname{Im}(\Gamma(\omega))$. The local energy dissipation rate is given by $\dot{\epsilon}_{1}=f_{1} u_{1} / 2$, where $u_{1}$ is the local velocity. Integrating the dissipation rate along the length of a tine of a tuning fork, we obtain

$$
\dot{E}=\int_{0}^{L} \dot{\epsilon}_{1} d l=\frac{\pi}{8} \rho \omega X^{2} L \xi U_{\mathrm{p}}^{2} \operatorname{Im}(\Gamma(\omega)),
$$

where $\xi$ again describes the velocity profile along the tine [32]. This leads to the drag force and drag coefficient:

$$
\begin{gathered}
F=\frac{\pi}{4} \rho \omega X^{2} L \xi U_{\mathrm{p}} \operatorname{Im}(\Gamma(\omega)), \\
C_{\mathrm{D}}=\frac{2 F}{\rho W L U_{\mathrm{p}}^{2}}=\frac{\pi \xi \omega X^{2} \operatorname{Im}(\Gamma(\omega))}{2 W U_{\mathrm{p}}} .
\end{gathered}
$$

To estimate the dissipation of a tuning fork of aspect ratio $A_{\mathrm{r}}=T / W$ in the high-Stokes-number limit, we express $\operatorname{Im}(\Gamma(\omega))$ as a function of the modified Stokes number $\beta_{d}$ :

$$
\lim _{\beta_{d} \rightarrow \infty} \operatorname{Im}\left(\Gamma\left(A_{\mathrm{r}}, \omega\right)\right)=c\left(A_{\mathrm{r}}\right) \beta_{d}^{-1 / 2}=\frac{2 c\left(A_{\mathrm{r}}\right)}{X} \sqrt{\frac{\nu}{\omega}},
$$

where $c\left(A_{\mathrm{r}}\right)$ is a constant coefficient for a given aspect ratio $A_{\mathrm{r}}$ that can be obtained with sufficient accuracy from the numerical data of Ref. [59].

Substituting for $\operatorname{Im}(\Gamma(\omega))$ in Eq. (A4), we get

$$
C_{\mathrm{D}}=\frac{\pi \xi X c\left(A_{\mathrm{r}}\right) \sqrt{\nu \omega}}{W U_{\mathrm{p}}}=\frac{\sqrt{2} \pi \xi X c\left(A_{\mathrm{r}}\right)}{W \operatorname{Re}_{\delta}},
$$

where $\operatorname{Re}_{\delta}=U_{\mathrm{p}} \delta / v$ is the boundary-layer-based Reynolds number (equivalent to the Donnelly number in superfluid $\mathrm{He}$ ). 
For both forks discussed here (and indeed for most tuning forks available), we have $T>W$ and therefore $X=T$ or, equivalently, $X / W=A_{\mathrm{r}}$ (in the opposite case we would have used $X=W$ ). The drag coefficient expressed for the normal component of superfluid helium then becomes $C_{\mathrm{D}}^{\mathrm{n}}=\Phi / \mathrm{Dn}$, where the prefactor $\Phi=\sqrt{2} \pi \xi A_{\mathrm{r}} c\left(A_{\mathrm{r}}\right)$ is again determined solely by the geometry of the tuning fork.

To evaluate $c\left(A_{\mathrm{r}}\right)$ for the custom-made fork of aspect ratio $A_{\mathrm{r}}=1.2$ and the commercial fork of aspect ratio $A_{\mathrm{r}}=2.1$, we analyze the results obtained for the aspect ratios of 1.0, 2.0, 5.0 as given in Ref. [59], obtaining $c(1.0) \approx 3.78, c(2.0) \approx$ 2.41 , and $c(5.0) \approx 1.57$. This gives by linear interpolation $c(1.2) \approx 3.51$ and $c(2.1) \approx 2.38$ for our tuning forks. Using $\xi=\frac{1}{4}$, we finally arrive at $C_{\mathrm{D}}^{\mathrm{n}} \simeq 4.67 / \mathrm{Dn}$ for the custommade fork and $C_{\mathrm{D}}^{\mathrm{n}} \simeq 5.55 / \mathrm{Dn}$ for the commercial one.

\section{APPENDIX B: DETERMINATION OF THE POSITION AND VELOCITY OF THE TORSIONALLY OSCILLATING DISK}

Sixteen black marks around the circumference of the disk are used to determine the deflection and angular velocity of the disk from recorded video sequences. The motion of the disk is recorded with a Casio EX-10 digital camera. The recordings are acquired at the frame rate of $240 \mathrm{fps}$ with a resolution of $512 \times 384$ pixels. A large optical lens is placed between the camera and the cryostat to improve the spatial resolution. Our raw data are in the form of video recordings of the motion of the disk during the experiments. Because the marks on the disk have rather low contrast to the not-entirely-uniform background, standard motion tracking software could not be used to process the videos. Hence, fairly complex postprocessing is required to extract quantitative and interpretable data.

The videos are split into individual frames and deinterlaced. The color images are converted to monochromatic bitmaps by dynamic contrast algorithms implemented in NI VISION software, so that the marks appear as black spots on a white background. These monochromatic bitmaps are then analyzed by a custom-made LABVIEW program. In the first pass, the program localizes the black areas in each image and evaluates their size and center of mass. In the second pass, using only numerical data from the first pass, it then links corresponding images of the same dot between all frames to each other (making special arrangements for those not reproduced in some of the bitmaps) and calculates the angular displacement of the disk in each instant. The program uses a self-calibration obtained from a complete revolution of the disk around its axis. The optical distortion from the lenses and the curved walls of the glass cryostat are negligible, as only a $10-\mathrm{mm}$ central portion of the field of view is used in the processing.

\section{APPENDIX C: HYDRODYNAMIC DESCRIPTION OF THE TORSIONALLY OSCILLATING DISK}

Here, we derive the equation of motion of the torsionally oscillating disk and the relevant hydrodynamic quantities. The motion of the harmonic torsional oscillator is given by the equation

$$
I_{0} \ddot{\varphi}+\kappa_{\mathrm{f}} \varphi=M_{\mathrm{F}},
$$

where $\varphi$ is the angular displacement, $I_{0}$ is the moment of inertia of the disk, $\kappa_{\mathrm{f}}$ is the moment of torsion of the fiber, and $M_{\mathrm{F}}$ represents the moment of drag forces due to the surrounding fluid.

In laminar flow, with some simplification, the moment of the frictional forces can be calculated on the basis of the analytical solution of the Navier-Stokes equations. First, we assume that the velocity profile $\boldsymbol{u}(\boldsymbol{r}, t)$ locally corresponds to the rotation of the rigid body modulated with the distance from the disk, $\boldsymbol{u}(\boldsymbol{r}, t)=\boldsymbol{\Omega}(z, t) \times \boldsymbol{r}$, where $\boldsymbol{\Omega}(z, t)=$ $(0,0, \Omega(z, t))$, in which $\Omega(z, t)$ is the instantaneous angular velocity of the fluid at the distance $z$ from the disk surface. Furthermore, we assume that the radius of the disk $R$ is significantly greater than its thickness $h_{\mathrm{d}}$ and all other relevant dimensions. The Navier-Stokes equation is then expressed in the form

$$
\frac{\partial \Omega(z, t)}{\partial t}=v \frac{\partial^{2} \Omega(z, t)}{\partial z^{2}},
$$

where $v$ is the kinematic viscosity. Assuming that any temporal changes of the amplitude of oscillation are much slower than one period of oscillation, the solution of this equation meeting the boundary conditions on the surface of the disk $(z=0)$ and at infinity can be expressed in the form

$$
\Omega(z, t)=\Omega_{0} e^{-z / \delta} e^{i(\omega t-z / \delta)},
$$

where $\Omega_{0}$ is the instantaneous amplitude of the disk's angular velocity and $\delta=\sqrt{2 v / \omega}$ is the viscous penetration depth. The total torque acting on the disk will be determined by integration of drag forces over both surfaces of the disk, neglecting the friction along its edge. The magnitude of the local viscous drag force $f_{\mathrm{L}}$ (per unit area) is given by $f_{\mathrm{L}}(r, t)=\eta \partial u(z, t) / \partial z$, where $\eta$ is the fluid dynamic viscosity. The magnitude of the local contribution to the torque of the viscous forces is then given as $M_{\mathrm{L}}(r, t)=r f_{\mathrm{L}}(r, t)$. The total moment of frictional forces is given as

$$
\begin{aligned}
M_{\mathrm{F}}(t) & =2 \int_{0}^{R} \int_{0}^{2 \pi} M_{\mathrm{L}}(r, t) r d \theta d r \\
& =-\pi \eta \frac{1+i}{\delta} \Omega_{0} R^{4} e^{i \omega t} \\
& =\frac{\pi}{\sqrt{2}}(1-i) \sqrt{\eta \omega \rho} \omega R^{4} \varphi_{0} e^{i \omega t},
\end{aligned}
$$

where $\Omega_{0} e^{i \omega t}=i \omega \varphi_{0} e^{i \omega t}$ was used, with $\varphi_{0}$ representing the instantaneous amplitude of angular displacement.

The moment of the friction forces is therefore phase shifted with respect to the angular velocity of the disk by $\pi / 4$. By defining a hydrodynamically induced moment of inertia $I_{\mathrm{HD}}=$ $\pi R^{4} \sqrt{\eta \rho / 2 \omega}$ and the coefficient $\Gamma=\pi R^{4} \sqrt{\eta \rho \omega / 2}$, we can rewrite the moment of the frictional forces as

$$
M_{\mathrm{F}}(t)=-\Gamma \dot{\varphi}(t)-I_{\mathrm{HD}} \ddot{\varphi}(t),
$$

where the two terms on the right-hand side correspond to dissipative and inertial torques, respectively. 
The energy dissipated by the viscous forces can be obtained as

$$
\begin{aligned}
\dot{E}(t) & =-2 \int_{0}^{R} \int_{0}^{2 \pi} \operatorname{Re}\left(M_{\mathrm{v}}(r, t)\right) \operatorname{Re}\left(\Omega_{t}\right) r d \theta d r \\
& =-\frac{\pi \eta \Omega_{0}^{2} R^{4}}{\delta}\left[\sin (\omega t) \cos (\omega t)-\cos ^{2}(\omega t)\right] .
\end{aligned}
$$

Averaging over one period, we get

$$
\langle\dot{E}\rangle=\frac{\pi}{2} \frac{\eta \Omega_{0}^{2} R^{4}}{\delta} .
$$

Using the fact that the total energy stored in the motion of the disk is $E=I_{0} \Omega_{0}^{2} / 2$, and its moment of inertia is given by $I_{0}=m R^{2} / 2$ (neglecting hydrodynamic contributions), we may define a fluidic quality factor

$$
\frac{1}{Q_{\mathrm{f}}}=\frac{\langle\dot{E}\rangle}{\omega E}=\frac{A}{m_{\mathrm{d}}} \sqrt{\frac{\eta \rho \omega}{2}},
$$

where $A=\pi R^{2}$ is the area of one side of the disk, and $m_{\mathrm{d}}$ is the disk's mass.

To define the drag coefficient, we follow the definition used in classical steady flow: the force $F$ acting on a body in steady flow is given by $F=\frac{1}{2} \mathrm{C}_{\mathrm{D}} A^{\prime} \rho U^{2}$, where $\mathrm{C}_{\mathrm{D}}$ is the dimensionless drag coefficient, $A^{\prime}$ is the cross section of the body perpendicular to the direction of motion, $\rho$ is the density of the fluid, and $U$ is the (homogeneous) velocity of the fluid. In analogy, it is possible to define the drag coefficient for the torsionally oscillating disk from

$$
\frac{M_{\mathrm{FD}}}{R}=\frac{1}{2} \mathrm{C}_{\mathrm{D}} A \rho \Omega_{0}^{2} R^{2},
$$

where $M_{\mathrm{FD}}=\Gamma \Omega_{0}$ is the dissipative part of the moment of frictional forces and we again use $A=\pi R^{2}$.

Finally, to define the dimensionless Donnelly number, we use the peak velocity at the circumference of the disk $U=$
$R \Omega_{0}$, yielding

$$
\mathrm{Dn}=\frac{R \Omega_{0} \rho_{\mathrm{n}} \delta_{\mathrm{n}}}{\eta} .
$$

Comparing with Eq. (C9), we arrive at $C_{D}^{n}=2 / D n$, where the normal component drag coefficient $\mathrm{C}_{\mathrm{D}}^{\mathrm{n}}$ differs from $\mathrm{C}_{\mathrm{D}}$ only by replacing the density $\rho$ with $\rho_{\mathrm{n}}$.

Substituting (C5) into the dynamic equation (C1) and dividing by the total moment of inertia $I=I_{0}+I_{\mathrm{HD}}$, we get

$$
\ddot{\varphi}+2 \gamma \dot{\varphi}+\omega_{0}^{2} \varphi=0,
$$

where $\gamma=\Gamma / 2 I$ is the damping coefficient and $\omega_{0}^{2}=\kappa_{\mathrm{f}} / I$ is the square of the intrinsic angular frequency of the undamped resonator. Thus, we have a standard equation of the damped harmonic oscillator, which is satisfied by the solution

$$
\varphi(t)=\varphi_{0} e^{-\gamma t} e^{i \omega t},
$$

where the angular frequency $\omega$ is related to the frequency of a hypothetical undamped oscillator by $\omega^{2}=\omega_{0}^{2}-\gamma^{2}$.

After processing the recorded videos of the disk motion, we obtain data in the form of $\varphi(t)$. From this, we determine the extrema $\varphi_{0, i}$ and the logarithmic decrements $\alpha_{i}=\ln \left(\varphi_{0, i-1}\right)-$ $\ln \left(\varphi_{0, i+1}\right)$, which are related to the damping coefficient $\gamma$ in Eq. (C11) by $\gamma_{i}=\alpha_{i} \omega /(2 \pi)$. The dissipative part of the moment of friction forces, the first term on the right-hand side of Eq. (C5), is then $M_{\mathrm{FD}, i}=2 I \omega \gamma_{i} \varphi_{0, i}$. The drag coefficient obtained from each experimental point may then be expressed as

$$
\mathrm{C}_{\mathrm{D}, i}=\frac{2 I \alpha_{i}}{\pi A \rho R^{3} \varphi_{0, i}} .
$$

If the hydrodynamic contribution to the moment of inertia is negligible, we may put $I \simeq I_{0}=m R^{2} / 2$, where the mass of the disk can be expressed as $m=A h_{\mathrm{d}} \rho_{\mathrm{d}}$, where $h_{\mathrm{d}}$ is the disk height and $\rho_{\mathrm{d}}$ its density. The drag coefficient can then be further simplified to

$$
\mathrm{C}_{\mathrm{D}, i}=\frac{1}{\pi} \frac{\rho_{\mathrm{d}} h_{\mathrm{d}} \alpha_{i}}{\rho R \varphi_{0, i}}
$$

which no longer requires the precise knowledge of $I$ or $I_{0}$.
[1] G. G. Stokes, On some cases of fluid motion, Trans. Cambridge Philos. Soc., Vol. VIII. Part I, 105 (1843), reprinted in: Mathematical and Physical Papers by G. G. Stokes, Vol. I, Cambridge University Press, 1880.

[2] G. H. Keulegan and L. H. Carpenter, J. Res. Natl. Bureau Standards 60, 423 (1958).

[3] C.-Y. Wang, J. Fluid Mech. 32, 55 (1968).

[4] T. Sarpkaya, J. Fluid Mech. 165, 61 (1986).

[5] M. Tatsuno and P. W. Bearman, J. Fluid Mech. 211, 157 (1990).

[6] K. L. Ekinci, V. Yakhot, S. Rajauria, C. Colosquiac, and D. M. Karabacak, Lab Chip 10, 3013 (2010).

[7] K. L. Ekinci, D. M. Karabacak, and V. Yakhot, Phys. Rev. Lett. 101, 264501 (2008).

[8] E. C. Bullard, J. Li, C. R. Lilley, P. Mulvaney, M. L. Roukes, and J. E. Sader, Phys. Rev. Lett. 112, 015501 (2014).
[9] M. Defoort, K. J. Lulla, T. Crozes, O. V. Maillet, O. Bourgeois, and E. Collin, Phys. Rev. Lett. 113, 136101 (2014).

[10] D. I. Bradley, R. George, A. M. Guénault, R. P. Haley, S. Kafanov, M. T. Noble, Y. A. Pashkin, G. R. Pickett, M. Poole, J. R. Prance, M. Sarsby, R. Schanen, V. Tsepelin, T. Wilcox, and D. E. Zmeev, Sci. Rep. 7, 4876 (2017).

[11] R. J. Donnelly and C. F. Barenghi, J. Phys. Chem. Ref. Data 27, 1217 (1998).

[12] R. D. McCarty, Thermophysical properties of helium-4 from 2 to $1500 \mathrm{~K}$ with pressures to 1000 atmospheres, Technical Note No. 631, National Bureau of Standards, 1972 (unpublished), https://nvlpubs.nist.gov/nistpubs/Legacy/ TN/nbstechnicalnote631.pdf.

[13] V. D. Arp and R. D. McCarty, The properties of critical helium gas, Technical Note 1334 (revised), National Bureau of 
Standards, 1998 (unpublished), https://nvlpubs.nist.gov/ nistpubs/Legacy/TN/nbstechnicalnote1334.pdf.

[14] S. Fuzier, B. Baudouy, and S. W. Van Sciver, Cryogenics 41, 453 (2001).

[15] B. Saint-Michel, E. Herbert, J. Salort, C. Baudet, M. Bon Mardion, P. Bonnay, M. Bourgoin, B. Castaing, L. Chevillard, F. Daviaud, P. Diribarne, B. Dubrulle, Y. Gagne, M. Gibert, A. Girard, B. Hébral, Th. Lehner, and B. Rousset, SHREK Collaboration, Phys. Fluids 26, 125109 (2014).

[16] J. J. Niemela, L. Skrbek, K. R. Sreenivasan, and R. J. Donnelly, Nature (London) 404, 837 (2000).

[17] L. D. Landau, J. Phys. (USSR) 5, 71 (1941); Zh. Eksp. Teor. Fiz. 11, 592 (1941) [J. Phys. (USSR) 11, 92 (1947)].

[18] R. J. Donnelly, Quantized Vortices in Helium II (Cambridge University Press, Cambridge, 1991).

[19] A. C. Hollis-Hallett, Proc. R. Soc. London, Ser. A 210, 404 (1952).

[20] R. J. Donnelly and A. C. Hollis-Hallett, Ann. Phys. 3, 320 (1958).

[21] E. L. Andronikashvili, Zh. Eksp. Teor. Fiz. 16, 780 (1946) [J. Phys. USSR 10, 201 (1946)].

[22] J. Jäger, B. Schuderer, and W. Schoepe, Phys. Rev. Lett. 74, 566 (1995).

[23] M. Niemetz and W. Schoepe, J. Low Temp. Phys. 135, 447 (2004).

[24] J. Luzuriaga, J. Low Temp. Phys. 108, 267 (1997).

[25] S. I. Davis, P. C. Hendry, and P. V. E. McClintock, Physica B (Amsterdam) 280, 43 (2000).

[26] H. A. Nichol, L. Skrbek, P. C. Hendry, and P. V. E. McClintock, Phys. Rev. Lett. 92, 244501 (2004).

[27] H. A. Nichol, L. Skrbek, P. C. Hendry, and P. V. E. McClintock, Phys. Rev. E 70, 056307 (2004).

[28] D. Charalambous, L. Skrbek, P. C. Hendry, P. V. E. McClintock, and W. F. Vinen, Phys. Rev. E 74, 036307 (2006).

[29] P. Švančara and M. La Mantia, J. Fluid Mech. 832, 578 (2017).

[30] R. Blaauwgeers, M. Blažková, M. Človečko, V. B. Eltsov, R. de Graaf, J. J. Hosio, M. Krusius, D. Schmoranzer, W. Schoepe, L. Skrbek, P. Skyba, R. E. Solntsev, and D. E. Zmeev, J. Low Temp. Phys. 146, 537 (2007).

[31] D. Garg, V. B. Efimov, M. Giltrow, P. V. E. McClintock, L. Skrbek, and W. F. Vinen, Phys. Rev. B 85, 144518 (2012).

[32] S. L. Ahlstrom, D. I. Bradley, M. Človečko, S. N. Fisher, A. M. Guénault, E. A. Guise, R. P. Haley, O. Kolosov, P. V. E. McClintock, G. R. Pickett, M. Poole, V. Tsepelin, and A. J. Woods, Phys. Rev. B 89, 014515 (2014).

[33] D. Schmoranzer, M. J. Jackson, V. Tsepelin, M. Poole, A. J. Woods, M. Človečko, and L. Skrbek, Phys. Rev. B 94, 214503 (2016).

[34] E. N. Martinez, P. Esquinazi, and J. Luzuriaga, Am. J. Phys. 58, 1163 (1990).

[35] C. L. Spiel, R. O. Pohl, and A. T. Zehnder, Rev. Sci. Instrum. 72, 1482 (2001).

[36] E. Zemma and J. Luzuriaga, J. Low Temp. Phys. 166, 171 (2012).

[37] D. Schmoranzer, M. Jackson, E. Zemma, and J. Luzuriaga, J. Low Temp. Phys. 187, 482 (2017).

[38] D. Duda, P. Švančara, M. La Mantia, M. Rotter, and L. Skrbek, Phys. Rev. B 92, 064519 (2015).
[39] W. F. Vinen, Proc. R. Soc. London, Ser. A 181, 1524 (1961).

[40] M. Morishita, T. Kuroda, A. Sawada, and T. Satoh, J. Low Temp. Phys. 76, 387 (1989).

[41] D. I. Bradley, D. O. Clubb, S. N. Fisher, A. M. Guénault, R. P. Haley, C. J. Matthews, G. R. Pickett, and K. L. Zaki, J. Low Temp. Phys. 138, 493 (2005).

[42] R. Goto, S. Fujiyama, H. Yano, Y. Nago, N. Hashimoto, K. Obara, O. Ishikawa, M. Tsubota, and T. Hata, Phys. Rev. Lett. 100, 045301 (2008).

[43] L. Skrbek and W. F. Vinen, The use of vibrating structures in the study of quantum turbulence, in Progress in Low Temperature Physics, edited by M. Tsubota and W. P. Halperin (Elsevier, Amsterdam, 2009), Vol. XVI, Chap. 4.

[44] L. Skrbek and W. F. Vinen, Proc. Natl. Acad. Sci. USA 111, 4699 (2014).

[45] C. F. Barenghi, L. Skrbek, and K. R. Sreenivasan, Proc. Natl. Acad. Sci. USA 111, 4647 (2014).

[46] R. J. Donnelly suggested using this dimensionless parameter, a "Reynolds number" based on the viscous penetration depth defined for the normal component of He II only, in his joint publication with A. C. Hollis-Hallett in Ref. [20] (note that such an analysis was not included in previous work of HollisHallett on the subject [19]). Unfortunately, the importance of this parameter was originally discounted, as it failed to describe the onset of turbulence for a sphere torsionally oscillating in He II. Now we know that this was because in the experiment the turbulent transition occurred first in the superfluid component and that the Donnelly number remains to be relevant for description of the normal fluid flow in this seminal boundary layer experiment.

[47] L. D. Landau and E. M. Lifshitz, Fluid Mechanics (Pergamon, London, 1959).

[48] W. I. Glaberson, W. W. Johnson, and R. M. Ostermeier, Phys. Rev. Lett. 33, 1197 (1974).

[49] R. Hänninen and W. Schoepe, J. Low Temp. Phys. 158, 410 (2010).

[50] M. Blažková, D. Schmoranzer, L. Skrbek, and W. F. Vinen, Phys. Rev. B 79, 054522 (2009).

[51] D. I. Bradley, M. J. Fear, S. N. Fisher, A. M. Guénault, R. P. Haley, C. R. Lawson, P. V. E. McClintock, G. R. Pickett, R. Schanen, V. Tsepelin, and L. A. Wheatland, J. Low Temp. Phys. 156, 116 (2009).

[52] A. W. Baggaley, J. Laurie, and C. F. Barenghi, Phys. Rev. Lett. 109, 205304 (2012).

[53] D. H. Wacks, A. W. Baggaley, and C. F. Barenghi, Phys. Rev. B 90, 224514 (2014).

[54] D. Schmoranzer, M. La Mantia, G. Sheshin, I. Gritsenko, A. Zadorozhko, M. Rotter, and L. Skrbek, J. Low Temp. Phys. 163, 317 (2011).

[55] D. I. Bradley, M. Človečko, S. N. Fisher, D. Garg, E. Guise, R. P. Haley, O. Kolosov, G. R. Pickett, V. Tsepelin, D. Schmoranzer, and L. Skrbek, Phys. Rev. B 85, 014501 (2012).

[56] K. Karrai and R. D. Grober, in Near-Field Optics, edited by M. A. Paesler and P. T. Moyer, Proceedings of the SPIE, Vol. 2535 (SPIE, Bellingham, WA, 1995), p. 69.

[57] S. Holt and P. Skyba, Rev. Sci. Instrum. 83, 064703 (2012).

[58] J. E. Sader, J. Appl. Phys. 84, 64 (1998). 
[59] D. R. Brumley, M. Willcox, and J. E. Sader, Phys. Fluids 22, 052001 (2010).

[60] A. M. Guénault, C. J. Kennedy, S. G. Mussett, and G. R. Pickett, J. Low Temp. Phys. 62, 511 (1986).

[61] K. J. Strnat, D. Li, and H. Mildrum, High and low temperature properties of sintered Nd-Fe-B magnets, in 8th International Workshop on Rare-Earth Magnets and their Applications, Dayton, OH, 1985; T. Hara, T. Tanaka, H. Kitamura, T. Bizen, X. Maréchal, T. Seike, T. Kohda, and Y. Matsuura, Phys. Rev. Accel. Beams 7, 050702 (2004).

[62] D. I. Bradley, P. Crookston, M. J. Fear, S. N. Fisher, G. Foulds, D. Garg, A. M. Guénault, E. Guise, R. P. Haley, O. Kolosov,
G. R. Pickett, R. Schanen, and V. Tsepelin, J. Low Temp. Phys. 161, 536 (2010).

[63] D. D. Awschalom, F. P. Milliken, and K. W. Schwarz, Phys. Rev. Lett. 53, 1372 (1984).

[64] A. P. Finne, T. Araki, R. Blaauwgeers, V. B. Eltsov, N. B. Kopnin, M. Krusius, L. Skrbek, M. Tsubota, and G. E. Volovik, Nature (London) 424, 1022 (2003).

[65] T. D. C. Bevan, A. J. Manninen, J. B. Cook, H. Alles, J. R. Hook, and H. E. Hall, J. Low Temp. Phys. 109, 423 (1997).

[66] J. T. Tough, Progress in Low Temperature Physics, Vol. VIII (North-Holland, Amsterdam, 1982).

[67] http://dx.doi.org/10.17635/lancaster/researchdata/264. 